\title{
Direct Numerical Simulations of $\mathrm{O}_{2} / \mathrm{H}_{2}$ Temporal Mixing Layers Under Supercritical Conditions
}

\author{
Nora Okong'o, ${ }^{*}$ Kenneth Harstad, ${ }^{\dagger}$ and Josette Bellan ${ }^{\ddagger}$ \\ Jet Propulsion Laboratory, California Institute of Technology, Pasadena, California 91 109-8099
}

\begin{abstract}
Direct numerical simulations of a supercritical oxygen/hydrogen temporal three-dimensional mixing layer are conducted to explore the features of high-pressure transitional mixing behavior. The conservation equations are formulated according to fluctuation-dissipation theory and are coupled to a modified Peng-Robinson equation of state. The boundary conditions are periodic in the streamwise and spanwise directions and of nonreflecting outflow type in the cross-stream direction. Simulations are conducted with initial Reynolds numbers of $6 \times 10^{2}$ and $7.5 \times 10^{2}$, initial pressure of $100 \mathrm{~atm}$, and temperatures of $400 \mathrm{~K}$ in the $\mathrm{O}_{2}$ and $600 \mathrm{~K}$ in the $\mathrm{H}_{2}$ stream. Each simulation encompasses the rollup and pairing of four initial spanwise vortices into a single vortex. The layer eventually exhibits distorted regions of high density-gradient-magnitude similar to the experimentally observed wisps of fluid at the boundary of supercritical jets. Analysis of the data reveals that the higher-Reynolds-number layer reaches transition, whereas the other one does not. The transitional layer is analyzed to elucidate its characteristics.
\end{abstract}

\section{Introduction}

$\mathbf{L}$ IQUID rocket propulsion relying on hydrogen/oxygen combustion is not a mature technology in that improvements in design are still necessary to mitigate efficiency and stability problems. To solve these problems in a systematic manner, it is required to understand the fundamental processes occurring in liquid rocket chambers. When liquid oxygen is injected in the chamber through one of the many ports, it encounters an environment in excess of its critical pressure, thereby becoming a supercritical fluid, that is, $\mathrm{O}_{2}$. We define a substance to be in a supercritical state when it is at a pressure $p$ or temperature $T$ exceeding its critical value (indicated here by a subscript $c$ ) (Refs. 1 and 2). What characterizesthe supercriticalstate is the impossibility of a two-phase region. Indeed, when the reduced pressure $p_{r} \equiv p / p_{c}>1$ or the reduced temperature $T_{r} \equiv T / T_{c}>1$, in the $(p, T)$ plane there is no longer the possibility of a two-phase, that is, gas/liquid, region, and instead there is only a single-phaseregion. The general term for the substance is fluid, that is, neither a gas nor a liquid. Noteworthy, because the critical locus of $\mathrm{O}_{2} / \mathrm{H}_{2}$ mixtures may include smaller or higher values of $\left(p_{c}, T_{c}\right)$ than those of the pure species, it is possible that locally in space and time the mixture could be at subcritical conditions, but such a situation cannot be assumed to persist for times comparable to those characteristic of mixing because of the continuous change in the mixture composition. Moreover, Harstad and Bellan ${ }^{3}$ concluded from their study of the evolution of an initial $\mathrm{O}_{2}$ fluid drop immersed in $\mathrm{H}_{2}$ at pressures from 6 to $40 \mathrm{MPa}$ that nowhere in the fluid drop or in the fluid mixture surrounding it are critical conditionsreached. In liquid rocket chambers the mean pressure is about $20 \mathrm{MPa}$, with peaks as high as $30 \mathrm{MPa}$, and therefore, both $\mathrm{O}_{2}$ and $\mathrm{H}_{2}$ are in a supercritical state (Table 1). Irrespective of the exact injection configuration, on entering the combustion chamber $\mathrm{O}_{2}$ disintegrates and mixes with $\mathrm{H}_{2}$ in a highly turbulent manner while being ignited, with ensuing combustion producing water and incomplete combustion products. From this description, it is immediately clear that $\mathrm{O}_{2}$ fluid disinte-

Received 13 March 2001; revision received 14 September 2001; accepted for publication 17 October 2001. Copyright (C) 2001 by the California Institute of Technology. Published by the American Institute of Aeronautics and Astronautics, Inc., with permission. Copies of this paper may be made for personal or internal use, on condition that the copier pay the $\$ 10.00$ per-copy fee to the Copyright Clearance Center, Inc., 222 Rosewood Drive, Danvers, MA 01923; include the code 0001-1452/02 \$10.00 in correspondence with the CCC.

*Scientist, Thermal and Propulsion Engineering, 4800 Oak Grove Drive. Drive.

Senior Engineer, Thermal and Propulsion Engineering, 4800 Oak Grove

${ }^{*}$ Senior Research Scientist, Thermal and Propulsion Engineering, 4800 Oak Grove Drive; josette.bellan@jpl.nasa.gov. gration plays a crucial role in determining the size of the $\mathrm{O}_{2}$ parcels entering in contact with $\mathrm{H}_{2}$ and in the efficiency of the combustion process.

This work is motivated by the need to study $\mathrm{O}_{2}$ fluid disintegration, although it is not immediately applicable to it and indeed represents only a first step toward this goal. $\mathrm{O}_{2}$ disintegration is a process essentially different from the much studied spray atomization that involves the breakup of a liquid into a multitude of drops. Liquid breakup relies on physical mechanisms involving the surface tension, and therefore it is an appropriate concept only when a surface tension does indeed exist. Recent experimental evidence from the $\mathrm{O}_{2} / \mathrm{H}_{2}$ observations of Mayer et al. ${ }^{4,5}$ indicates that $\mathrm{O}_{2}$ disintegration assumes characteristics different from atomization and that these characteristics reflect features associated with supercritical conditions. In contrast to the process of atomization, past the critical point of the fluid, disintegration assumes the aspect of what Chehroudi et al. ${ }^{6}$ call "fingers" or "comb-like structures" at transcritical conditions, having an increasingly gaseous appearance with increasing pressure. Their experiments were conducted with $\mathrm{N}_{2} / \mathrm{N}_{2}$, $\mathrm{N}_{2} /\left(\mathrm{CO}+\mathrm{N}_{2}\right), \mathrm{He} / \mathrm{N}_{2}$ and $\mathrm{O}_{2} / \mathrm{N}_{2}$. Raman scattering measurements of the radialdensity in free $\mathrm{N}_{2}$ jets at $4 \mathrm{MPa}$ by Oschwald and $\mathrm{Schik}^{7}$ show sharp profiles independent of the injection temperature, indicating the occurrence of sharp density gradients. These regions of sharp density gradients are indeed one of the distinctive optical features in environments at supercritical conditions. Not only have they been experimentally observed, but they have also been identified in temporal simulations of supercritical heptane/nitrogen three-dimensionalmixing layers ${ }^{8,9}$; analysis of an enlarged database of Miller et al. ${ }^{8}$ by Okong'o and Bellan ${ }^{10}$ revealed that these regions of large density-gradient-magnitude play a crucial role in delaying transition to turbulence by acting similar to material surfaces in that they damp emerging turbulent eddies. Although mixing layers are different from jets, the jet mixing region behaves essentially as a mixing layer, making mixing layer simulations pertinent to jets. Moreover, Metcalfe et al. ${ }^{11}$ point out and discuss the qualitative similarities between optical laboratory observations and temporal simulations. The important factor is that both experiments and theory are for fluids at supercritical pressures.

Because the behavior of a binary species system depends on the identity of the species, it is uncertainif our previous findings for heptane/nitrogen are immediately applicable to the oxygen/hydrogen system, which is of interest here. For example, Harstad and Bellan ${ }^{12}$ found in drop studies that under supercritical conditions, compared to the heptane/nitrogen combination, the $\mathrm{O}_{2} / \mathrm{H}_{2}$ system displays an increased solubility and also much larger effective Lewis numbers $L e_{\text {eff }}$. The increased solubility results from the thermodynamic mixing rules, ${ }^{2}$ whereas the enhanced effective Lewis numbers were attributed to the considerably larger difference of the specific, that 
Table 1 Pure species properties

\begin{tabular}{lrccccr}
\hline \hline Species & $m, \mathrm{~g} / \mathrm{mol}$ & $T_{c}, \mathrm{~K}$ & $p_{c}, \mathrm{MPa}$ & $v_{c}, \mathrm{~cm}^{3} / \mathrm{mol}$ & $Z_{c}$ & \multicolumn{1}{c}{$\Omega$} \\
\hline Hydrogen & 2.0159 & 33.0 & 1.2838 & 64.284 & 0.306 & -0.216 \\
Oxygen & 31.9988 & 154.58 & 5.0430 & 73.368 & 0.288 & 0.025 \\
\hline \hline
\end{tabular}

is, mass-based, enthalpies of the components in the $\mathrm{O}_{2} / \mathrm{H}_{2}$ system compared to the equivalent difference for heptane/nitrogen.

The present paper is devoted to the study of supercritical $\mathrm{O}_{2} / \mathrm{H}_{2}$ three-dimensional mixing layers through direct numerical simulations (DNS) as a means of obtaining information about its specific fundamental behavior. This represents only the first step toward numerical studies of $\mathrm{O}_{2} / \mathrm{H}_{2}$ under liquid rocket chamber conditions. Those studies are more arduous due to the much larger density stratification. Because in DNS all scales of the flow are resolved, these types of simulations appear ideal for developing information to be used eventually in modeling $\mathrm{O}_{2}$ disintegration, as well as turbulent $\mathrm{O}_{2} / \mathrm{H}_{2}$ mixing. In Sec. II, we briefly recall the conservation equations derived elsewhere. ${ }^{8,9}$ Section III is devoted to describing the particular aspects of the equation of state (EOS) implemented in the model to obtain increased accuracy with respect to the typical Peng-Robinson formalism. In Sec. IV, we address the choice of the transport coefficients. The configuration and boundary conditions are addressed in Sec. V, whereas, in Sec. VI, we discuss the numerics. Section VII is focused on the initial conditions and results are presented from a linear, inviscid stability analysis, which is used to understand specific aspects of the $\mathrm{O}_{2} / \mathrm{H}_{2}$ mixing layer that are necessary for choosing initial conditionsfor the three-dimensionalsimulations. In Sec. VIII we present two three-dimensionalsimulations at differentinitial Reynolds numbers $R e_{0}$. The global characteristics of the layers combined with information from energy spectra show that only the larger Reynolds number $R e_{0}$ layer reaches transition. To understand the characteristics of the transitional state, we concentrate on detailed studies of the layer with the larger Reynolds number $R e_{0}$ and present the results of this analysis. A summary and conclusions appear in Sec. IX.

\section{Conservation Equations}

The conservation equations are briefly recalled, and the reader is referred for details to Miller et al., ${ }^{8}$ Okong'o and Bellan, ${ }^{9}$ and Okong'o and Bellan. ${ }^{13}$ For the binary mixture under consideration, the conservation equations are

$$
\begin{gathered}
\frac{\partial \rho}{\partial t}+\frac{\partial\left(\rho u_{j}\right)}{\partial x_{j}}=0 \\
\frac{\partial\left(\rho u_{i}\right)}{\partial t}+\frac{\partial\left(\rho u_{i} u_{j}+p \delta_{i j}\right)}{\partial x_{j}}=\frac{\partial \tau_{i j}}{\partial x_{j}} \\
\frac{\partial\left(\rho Y_{\mathrm{O}}\right)}{\partial t}+\frac{\partial\left(\rho Y_{\mathrm{O}} u_{j}\right)}{\partial x_{j}}=-\frac{\partial j_{\mathrm{O} j}}{\partial x_{j}} \\
\frac{\partial\left(\rho e_{t}\right)}{\partial t}+\frac{\partial\left[\left(\rho e_{t}+p\right) u_{j}\right]}{\partial x_{j}}=-\frac{\partial q_{\mathrm{IK} j}}{\partial x_{j}}+\frac{\partial \tau_{i j} u_{i}}{\partial x_{j}}
\end{gathered}
$$

where $x$ is a Cartesian coordinate; $t$ is time; $\rho$ is the density; $u_{i}$ is the velocity; $e_{t}=e+u_{i} u_{i} / 2$ is the total energy, that is, internal energy $e$ plus kinetic energy; $p$ is the thermodynamic pressure; and $Y_{\mathrm{O}}$ is the mass fraction of $\mathrm{O}_{2}$. Furthermore, $\boldsymbol{q}_{\mathrm{IK}}$ is the Irwing-Kirkwood (IK) form of the heat flux vector (see Sarman and Evans ${ }^{14}$ ), $j_{\mathrm{O}}$ is the $\mathrm{O}_{2}$ mass flux vector, and $\tau_{i j}$ is the Newtonian viscous stress tensor

$$
\tau_{i j}=\mu\left[\frac{\partial u_{i}}{\partial x_{j}}+\frac{\partial u_{j}}{\partial x_{i}}-\frac{2}{3} \frac{\partial u_{k}}{\partial x_{k}} \delta_{i j}\right]
$$

where $\delta_{i j}$ is the Kronecker delta function and $\mu$ is the mixture viscosity that is in general a function of the thermodynamic state variables. The species mass flux and heat flux are given by

$$
j_{\mathrm{O} j}=-\left[j_{\mathrm{O} j}^{\prime}+\left(\alpha_{\mathrm{IK}}-\alpha_{h}\right) Y_{\mathrm{O}} Y_{\mathrm{H}} \frac{\rho D}{T} \frac{\partial T}{\partial x_{j}}\right]
$$

$$
\begin{gathered}
q_{\mathrm{IK} j}=-\lambda_{\mathrm{IK}}^{\prime} \frac{\partial T}{\partial x_{j}}-\alpha_{\mathrm{IK}} R_{u} T \frac{m}{m_{\mathrm{O}} m_{\mathrm{H}}} j_{\mathrm{O} j}^{\prime} \\
j_{\mathrm{O} j}^{\prime}=\rho D\left[\alpha_{D} \frac{\partial Y_{\mathrm{O}}}{\partial x_{j}}+\frac{Y_{\mathrm{O}} Y_{\mathrm{H}}}{R_{u} T} \frac{m_{\mathrm{O}} m_{\mathrm{H}}}{m}\left(\frac{v_{, \mathrm{O}}}{m_{\mathrm{O}}}-\frac{v_{\mathrm{H}}}{m_{\mathrm{H}}}\right) \frac{\partial p}{\partial x_{j}}\right]
\end{gathered}
$$

with

$$
\alpha_{h}=\frac{1}{R_{u} T} \frac{m_{\mathrm{O}} m_{\mathrm{H}}}{m}\left(\frac{h_{, \mathrm{O}}}{m_{\mathrm{O}}}-\frac{h_{, \mathrm{H}}}{m_{\mathrm{H}}}\right)
$$

The notationin Eqs. (6-9) is as follows: $T$ is the temperature; $D$ is the binary diffusion coefficient; $\alpha_{D}$ is the mass diffusion factor, which is a thermodynamic quantity; the mass fraction of $\mathrm{H}_{2}$ is $Y_{\mathrm{H}}=1-Y_{\mathrm{O}}$; $m_{\alpha}$ is the molar mass of species $\alpha ; m=m_{\mathrm{O}} X_{\mathrm{O}}+m_{\mathrm{H}} X_{\mathrm{H}}$ is the mixture molar mass, where the molar fraction $X_{\alpha}=m Y_{\alpha} / m_{\alpha}$; $v_{, \alpha}=\left(\partial v / \partial X_{\alpha}\right)_{T, p, X_{\beta}(\beta \neq \alpha)}$ is the partial molar volume; $h_{, \alpha}=(\partial h /$ $\left.\partial X_{\alpha}\right)_{T, p, X_{\beta}(\beta \neq \alpha)}$ is the partial molar enthalpy; $v=X_{\mathrm{H}} v_{, \mathrm{H}}+X_{\mathrm{O}} v_{, \mathrm{O}}$ is the molar volume related to the density by $v=m / \rho ; h=X_{\mathrm{H}} h_{\mathrm{H}_{\mathrm{H}}}+$ $X_{\mathrm{O}} h_{, \mathrm{O}}$ is the molar enthalpy; $R_{u}$ is the universal gas constant; and $\lambda_{\text {IK }}^{\prime}$ is a thermal conductivity defined from the transport matrix,

$$
\lambda_{\mathrm{IK}}^{\prime}=\lambda+X_{\mathrm{H}} X_{\mathrm{O}} \alpha_{\mathrm{IK}} \alpha_{\mathrm{BK}} R_{u} \rho D / m
$$

where $\lim _{p \rightarrow 0} \lambda=\lambda_{\mathrm{KT}}$, as discussed in Ref. 15, where the subscript KT refers to kinetic theory. The new transport coefficients associated with the Soret (in the molar fluxes) and the Dufour (in the heat flux) terms of the transport matrix are $\alpha_{\mathrm{BK}}$ and $\alpha_{\mathrm{IK}}$, which are the thermal diffusion factors corresponding to the Bearman-Kirkwood (BK) and the IK forms of the heat flux (see Sarman and Evans ${ }^{14}$ ). These transport coefficients are characteristic of each of the particular species pairs, and they obey the relationship

$$
\alpha_{\mathrm{BK}}=\alpha_{\mathrm{IK}}-\alpha_{h}
$$

as shown by Harstad and Bellan. ${ }^{15}$ Additionally, $\lim _{p \rightarrow 0} \alpha_{\mathrm{IK}} \neq \alpha_{\mathrm{KT}}$ and $\lim _{p \rightarrow 0} \alpha_{\mathrm{BK}}=\alpha_{\mathrm{KT}}$.

To solve the preceding system of equations, it must be closed by specifying the EOS and the transport properties.

\section{Equation of State}

The pressure can be calculated from the well-known PengRobinson (PR) EOS given the PR molar volume $v_{\mathrm{PR}}$ as

$$
p=\frac{R_{u} T}{\left(v_{\mathrm{PR}}-b_{m}\right)}-\frac{a_{m}}{\left(v_{\mathrm{PR}}^{2}+2 b_{m} v_{\mathrm{PR}}-b_{m}^{2}\right)}
$$

where $a_{m}$ and $b_{m}$ are functions of $T$ and $X_{\alpha}$ (Appendix). Because of the inaccuracy of the PR EOS at high pressures (see Ref. 16), $v_{\mathrm{PR}}$ may differ significantly from the actual molar volume $v$. Therefore, for improved accuracy, we use a modified PR EOS in which both $v_{\mathrm{PR}}$ and the volume shift

$$
v_{S}=v-v_{\mathrm{PR}}
$$

are calculated from the PR EOS given $p, T$, and $X_{\alpha}$, as shown subsequently.

All of the thermodynamic properties such as the molar enthalpy $h=G-T(\partial G / \partial T)_{p, X}$, the constant-pressure molar heat capacity $C_{p}=(\partial h / \partial T)_{p, X}$, and the speed of sound $a_{s}=1 / \sqrt{ }\left(\rho \kappa_{s}\right)$ are calculated in a consistent manner from the same EOS using the Gibbs energy $G$ :

$$
\begin{aligned}
& G\left(T, p, X_{\alpha}\right)=\int_{v}^{v_{u}} p\left(v^{\prime}, T, X_{\alpha}\right) \mathrm{d} v^{\prime}+p v-R_{u} T \\
& \quad+\sum_{\alpha} X_{\alpha}\left[G_{\alpha}^{0}+R_{u} T \ln \left(X_{\alpha}\right)\right]
\end{aligned}
$$

where the superscript 0 represents the low-pressure reference condition for the integration as generally used in the departure function formalism described by Reid et al. ${ }^{16}$ The integral is ill defined for a zero pressure reference condition; hereinafter we choose $p^{0}=1$ bar such that $v_{u}=R_{u} T / p^{0}$. The volume shift $v_{S}$ is calculated from $G_{\alpha}^{0}$ 
(found by curve fits to accurate pure substance enthalpy and entropy values; see Harstad et al. ${ }^{17}$ ) as

$$
v_{S}=\sum X_{\alpha} \frac{\partial G_{\alpha}^{0}}{\partial p}
$$

The isentropic compressibility $\kappa_{s}$ is calculated from

$$
\kappa_{s}=\kappa_{T}-v T \alpha_{v}^{2} / C_{p}
$$

where the expansivity $\alpha_{v}$ and the isothermal compressibility $\kappa_{T}$ are given by

$$
\alpha_{v}=-\frac{(\partial p / \partial T)_{v, X}}{v(\partial p / \partial v)_{T, X}}, \quad \kappa_{T}=\frac{-1}{v(\partial p / \partial v)_{T, X}}
$$

The mass diffusion factor $\alpha_{D}$ is calculated from the fugacity coefficients $\varphi_{\alpha}$ (which are related to the Gibbs energy), through

$$
\alpha_{D}=1+X_{\alpha} \frac{\partial \ln \left(\varphi_{\alpha}\right)}{\partial X_{\alpha}}
$$

and portrays departures from mixture ideality, that is, $\alpha_{D}=1$. Note that $\alpha_{D}$ is independent of which of the two species is chosen in the calculation of Eq. (18).

These equations specify the entire thermodynamics of the binary mixture.

\section{Transport Coefficients}

Based on contour plots (not shown) using accurate species transport properties calculated as by Harstad and Bellan, ${ }^{18}$ the following correlations were obtained for the Schmidt number $S c$ and the Prandtl number $P r$ in the range 200-800 K:

$$
\begin{aligned}
& \mu= \mu_{R}\left\{T /\left[\left(T_{1}+T_{2}\right) / 2\right]\right\}^{0.75} \\
& S c \equiv\left(\mu / \rho \alpha_{D} D\right)=\left(1.334-0.668 Y_{\mathrm{O}}-0.186 Y_{\mathrm{O}}^{2}-0.268 Y_{\mathrm{O}}^{6}\right) \\
& \times\left[1+(88.6 / T)^{1.5}\right] \\
& \operatorname{Pr} \equiv \mu C_{p} /(m \lambda)=1.335 / T^{0.1}
\end{aligned}
$$

where $T$ is in Kelvin, $\mu_{R}$ is a reference viscosity, and the reference temperatures $T_{1}$ (upper $\mathrm{H}_{2}$ stream) and $T_{2}$ (lower $\mathrm{O}_{2}$ stream) correspond to the freestream temperatures for mixing layer simulations.

The value of the reference viscosity is determined by the specified value of Reynolds number $R e_{0}$ (to be discussed).

The thermal diffusionfactor is selected as by Harstad and Bellan ${ }^{18}$ with $\alpha_{\mathrm{BK}}=0.2$, and $\alpha_{\mathrm{IK}}$ is calculated from Eq. (11). The sensitivity of the solution to the value of the thermal diffusion factor was assessed by Harstad and Bellan ${ }^{3}$ for $\mathrm{O}_{2}$ drops in $\mathrm{H}_{2}$, and it has been determined to be minor at $10 \mathrm{MPa}$, which is approximately the freestream pressure in the present simulations. In the realm of turbulent mixing layers, Miller et al. ${ }^{8}$ have explored the impact of the $\alpha_{\mathrm{BK}}$ and $\alpha_{\mathrm{IK}}$ values on the solution for heptane/nitrogen and have shown that for small values, such as those in the present calculations, the influence is minor.

\section{Configuration and Boundary Conditions}

The temporally developing mixing layer configuration is shown in Fig. 1, which shows the definition of the streamwise, $x_{1}$, crossstream, $x_{2}$, and spanwise, $x_{3}$, coordinates. The layer is not symmetric in extent in the $x_{2}$ direction because it was found in our simulations that the layer growth is considerably larger in the hydrogen side. The freestream density, $\rho_{1}$ or $\rho_{2}$, is calculated for each pure species at its freestream temperature, $T_{1}$ or $T_{2}$, and at the initial uniform pressure $p_{0}$. The vorticity thickness is defined as $\delta_{\omega}(t)=\Delta U_{0} /\left(\partial\left\langle u_{1}\right\rangle / \partial x_{2}\right)_{\max }$, where $\left\langle u_{1}\right\rangle$ is the $x_{1}-x_{3}$ planar average velocity in the streamwise direction and $\Delta U_{0}=U_{1}-U_{2}$ is the velocity difference across the layer. Miller et al. ${ }^{8}$ explain the choice of the velocities of the two streams in a simulation initiated
Fig. 1 Mixing layer configuration.

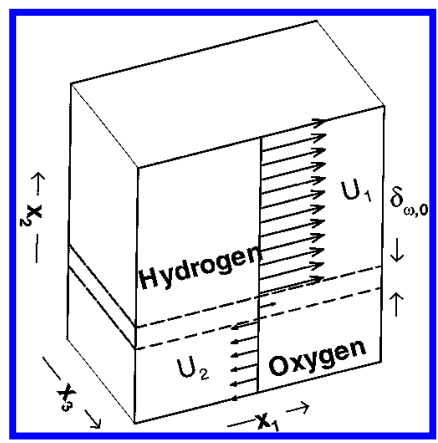

with four streamwise vortices pairing twice to produce an ultimate vortex. The choice of $U_{1}$ and $U_{2}$ for a real fluid

$$
\begin{gathered}
U_{1}=2 M_{c, 0} a_{s_{1}}\left[1+\left(a_{s_{1}} / a_{s_{2}}\right) \sqrt{\left(\rho_{1} Z_{1}\right) /\left(\rho_{2} Z_{2}\right)}\right]^{-1} \\
U_{2}=-\sqrt{\left(\rho_{1} Z_{1}\right) /\left(\rho_{2} Z_{2}\right)} U_{1}
\end{gathered}
$$

was made with the intent of keeping the ultimate vortex stationary in the computational domain. $M_{c, 0}$ is the convective Mach number and $Z=p /\left(\rho T R_{u} / m\right)$ is the compression factor indicating departures from the perfect gas behavior, that is, $Z=1$. The specification of $M_{c, 0}$, therefore, determines $\Delta U_{0}$. Given the initial streamwise velocity profile $u_{1}$ based on $U_{1}$ and $U_{2},\left(\partial\left\langle u_{1}\right\rangle / \partial x_{2}\right)_{\max }$ and, hence, $\delta_{\omega, 0} \equiv \delta_{\omega}(0)$ are calculated.

The specified value of the initial flow Reynolds number,

$$
\operatorname{Re}_{0}=\frac{0.5\left(\rho_{1}+\rho_{2}\right) \Delta U_{0} \delta_{\omega, 0}}{\mu_{R}}
$$

is used to calculate $\mu_{R}$.

The boundary conditions are periodic in the streamwise and spanwise directions and of nonreflecting outflow type for real gas as derived by Okong'o and Bellan. ${ }^{13}$ The outflow-type conditions are essential to maintain stability because the initial perturbation causes large pressure waves that must be allowed out of the domain with minimal reflection.

\section{Numerics}

\section{A. General Method}

The conservationequations are numerically solved using a fourthorder explicit Runge-Kutta time integration and a sixth-order compact scheme for spatial derivatives (see Ref. 19). Time stability is achieved by filtering the conservativevariables every five time steps in the interior, in each spatial direction alternately, using an eighthorder filter. ${ }^{19}$ Because high-order boundary filters are unstable, no filtering is applied at the nonperiodic $x_{2}$ boundaries. The computations were parallelized using three-dimensional domain decomposition and message passing. The tridiagonal solver for the compact derivative scheme was efficiently parallelized using the method in Ref. 20. The simulations were performed on an SGI Origin 2000 supercomputer, using 64 processors.

In our solution protocol, once the pressure, temperature, and mole fractions are specified, the density and energy are calculated from the EOS. To calculate the pressure and temperature from the known energy, mole fractions, and molar volume, we iterate at each time step, as described next.

\section{B. Iterative Solution for the Pressure and Temperature}

When the modified PR EOS is used ${ }^{17}$ once $p, T$, and $X_{\alpha}$ are specified, one can calculate $\rho, e$, and $Y_{\alpha}$. However, in the DNS the dependent variables are $\rho, e$, and $Y_{\alpha}$ from which $v$ and $X_{\alpha}$ are calculated as

$$
m=\frac{1}{\sum_{\alpha}\left(Y_{\alpha} / m_{\alpha}\right)}, \quad X_{\alpha}=\frac{m Y_{\alpha}}{m_{\alpha}}, \quad v=\frac{m}{\rho}
$$

This means that an iteration is necessary to obtain the values of $p$ and $T$ corresponding to the DNS $\rho$ and $e$. The procedure for this iteration is to update $p$ and $T$ at iteration level $n,\left(p^{n}\right.$ and $\left.T^{n}\right)$, using 


$$
\begin{gathered}
T^{n}=T^{n-1}+\mathrm{d} T, \quad p^{n}=p^{n-1}+\mathrm{d} p \\
\mathrm{~d} T=\left(\frac{\partial T}{\partial v}\right)_{e, X_{\alpha}}^{n-1} \mathrm{~d} v+\left(\frac{\partial T}{\partial e}\right)_{v, X_{\alpha}}^{n-1} \mathrm{~d} e+\sum_{\alpha}\left(\frac{\partial T}{\partial X_{\alpha}}\right)_{\substack{v, e, X_{\beta} \\
\beta \neq \alpha}}^{n-1} \mathrm{~d} X_{\alpha} \\
\mathrm{d} p=\left(\frac{\partial p}{\partial v}\right)_{v, X_{\alpha}}^{n-1} \mathrm{~d} v+\left(\frac{\partial p}{\partial e}\right)_{v, X_{\alpha}}^{n-1} \mathrm{~d} e+\sum_{\alpha}\left(\frac{\partial p}{\partial X_{\alpha}}\right)_{\substack{v, e, X_{\beta} \\
\beta \neq \alpha}}^{n-1} \mathrm{~d} X_{\alpha}
\end{gathered}
$$

Because $X_{\alpha}$ are known, $\mathrm{d} X_{\alpha}=0$. Also,

$$
\mathrm{d} e=e-e^{n-1}, \quad \mathrm{~d} v=v-v^{n-1}
$$

where

$$
e^{n-1}=e\left(p^{n-1}, T^{n-1}, X_{\alpha}\right), \quad v^{n-1}=v\left(p^{n-1}, T^{n-1}, X_{\alpha}\right)
$$

are computed from the EOS. The needed derivatives can be calculated from $C_{p}, \kappa_{T}$, and $\alpha_{v}$ (where $e$ is in joules per kilogram and $C_{p}$ and $C_{v}$ are in joules per mol Kelvin) as

$$
\begin{aligned}
& \left(\frac{\partial T}{\partial v}\right)_{e, X_{\alpha}}=\frac{1}{v \alpha_{v}}\left(1-\frac{C_{p}}{C_{v}}\right)+\frac{p}{C_{v}}, \quad\left(\frac{\partial T}{\partial e}\right)_{v, X_{\alpha}}=\frac{m}{C_{v}} \\
& \left(\frac{\partial p}{\partial v}\right)_{e, X_{\alpha}}=\frac{1}{\kappa_{T}}\left(-\frac{C_{p}}{C_{v}} \frac{1}{v}+\alpha_{v} \frac{p}{C_{v}}\right), \quad\left(\frac{\partial p}{\partial e}\right)_{v, X_{\alpha}}=\frac{m}{C_{v}} \frac{\alpha_{v}}{\kappa_{T}}
\end{aligned}
$$

The iteration is conducted by updating $p$ and $T$ until $\mathrm{d} e$ and $\mathrm{d} v$ are within a desired tolerance, for example, $\mathrm{d} e / e<10^{-6}$ and $\mathrm{d} v / v<$ $10^{-6}$. The initial guess for $p$ and $T$ is from the previous time step or the previous Runge-Kutta stage. For the $\mathrm{O}_{2} / \mathrm{H}_{2}$ regime under consideration, $p$ and $T$ converge in two iterations. It was found that, if the initial guess for $p$ and $T$ is $\left[p_{0},\left(T_{1}+T_{2}\right) / 2\right]$, four or five iterations are required for convergence. Thus, there appears to be no need to store $p$ and $T$, therefore relaxing some of the computation overhead associated with this iteration. In fact, the memory overhead of the iterative method is the addition of the four arrays necessary to store $e, v, e^{n-1}$, and $v^{n-1}$. Compared with the heptane/nitrogen simulations where a PR EOS for computing the pressure and an energy fit for temperature were employed, ${ }^{9}$ here an additional $50 \%$ CPU time per time step is used. However, for the $\mathrm{O}_{2} / \mathrm{H}_{2}$ mixture, the form of the energy fit for the temperature would be considerably more complicated than that of the heptane/nitrogen mixture, and the pressure can no longer be computed directly from the molar volume.

\section{Resolution}

The appropriateresolution of all scales is checked in several ways. First, visual inspection of the dilatation field $\nabla \cdot \boldsymbol{u}$, which is the most sensitive to numerical errors, indicates absence of small-scale fluctuations in $\nabla \cdot \boldsymbol{u}$; this absence is a well-known indicator of good resolution. Moreover, plots of streamwise and spanwise energy spectra of the velocity fluctuations (not shown) display more than a 10 order of magnitude ratio between the large and the small scales, indicating that most of the energy is in the large scales and that there is no accumulation of energy at small scales. Finally, the insensitivity of the solution to the resolution has been proved in two-dimensional simulations with the same species system and with the same freestream conditions.

Generally, the flowfield is extremely sensitive to having an appropriateresolution, and its lack is manifested by the code crashing. Another diagnostic of inadequate resolution is an increasing number of iterations for the convergence of the calculation involving the EOS, leading eventually to the code crashing as well.

\section{Initial Conditions}

The appropriate initial conditions for simulating the evolution of mixing layers are notoriously difficult to choose, especially for density stratified situations. (See a discussion by Miller et al. ${ }^{8}$ ) To this end, following Drazin and Reid, ${ }^{21}$ there are two issues that must be addressed: First, one must inquire about the mean flow and, second, about the appropriate disturbance. Both of these issues were thoroughly investigated by Okong'o and Bellan ${ }^{22}$ for real gases and applied to the heptane/nitrogen system under supercritical conditions. The same formalism is applied here to explore the specific features of the $\mathrm{O}_{2} / \mathrm{H}_{2}$ system. Having determined here (by numerically solving the laminar equations) that the form of the mean flow for the $\mathrm{O}_{2} / \mathrm{H}_{2}$ system is that of an error function like profile (not shown) and, thus, that it has a single inflection point, a two-dimensional stability analysis is performed with the error function representing the mean flow. According to Drazin and Reid, ${ }^{21}$ this is acceptable for mean flows displaying a single inflection point because the stability analysis is not sensitive to the exact form of the mean flow.

Ideally, we would like to perform simulations in the region of low $\mathrm{O}_{2}$ temperatures, for example, $90 \mathrm{~K}$, and high $\mathrm{H}_{2}$ temperatures, for example, larger than $1000 \mathrm{~K}$, characteristic of liquid oxygen injection in the combustion chamber. However, simple thermodynamic considerations show that, given current computational capabilities, it is impossible to conduct DNS under such conditions. The density ratio vs temperature for $\mathrm{O}_{2} / \mathrm{H}_{2}$ in the range of low (but still higher than $90 \mathrm{~K}$ ) $\mathrm{O}_{2}$ temperatures and $\mathrm{H}_{2}$ temperatures up to $320 \mathrm{~K}$ (and, therefore, much lower than those in the combustion chamber) at two relevant pressures are shown in Fig. 2a. Even for these conditions that do not offer the desired temperature ratio, the density stratification is well beyond those that are currently computable in threedimensional configurations (see subsequent discussion on the computationalresolutionfor lesserstratification). As $T_{2}$ decreases, $\rho_{2} / \rho_{1}$

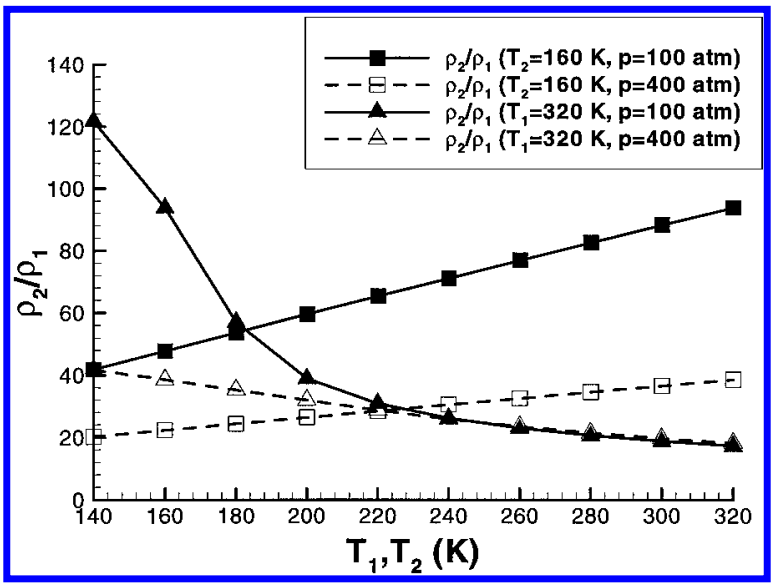

Fig. 2a Ratio $\rho_{2} / \rho_{1}$ vs $T_{1}$ and $T_{2}$ for $\mathrm{O}_{2} / \mathrm{H}_{2}$ at different conditions.

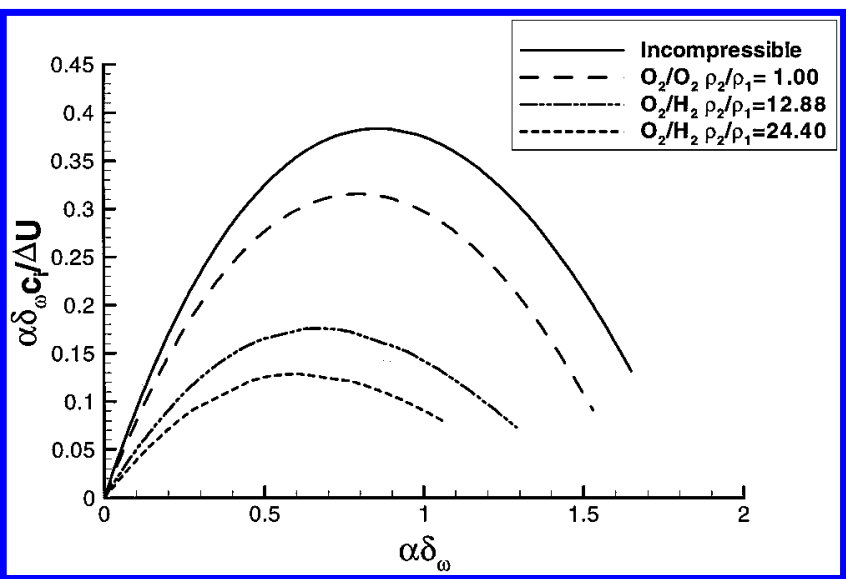

Fig. 2b Stability curve for $\mathrm{O}_{2} / \mathrm{O}_{2}$ and $\mathrm{O}_{2} / \mathrm{H}_{2}$ at $p=100$ atm at several values of $\rho_{2} / \rho_{1}$. 
becomes larger, leading to increasing computational constraints associated with an increased number of nodes in the direction of the initial density gradient stratification, that is, $x_{2}$, for the same resolution. Therefore, to enable resolution of all scales, the $\mathrm{O}_{2} / \mathrm{H}_{2}$ DNS must be restricted to relatively small ratios $\left|T_{2}-T_{1}\right| / T_{2}$.

A standard stability analysis is, therefore, performed for several mean flow conditions as listed in Tables 2-4, with the freestream velocity specified by

$$
\bar{u}_{1}(\infty)=U_{1}, \quad \bar{u}_{1}(-\infty)=U_{2}
$$

and the mean velocity, temperature, and mass fraction following an error function profile. ${ }^{22}$ Because the pressure is constant, the speed of sound and inverse of the density also assume approximately error function profiles. The stability curves associated with the mean flow conditions are shown in Fig. $2 b$, with the $\mathrm{O}_{2} / \mathrm{O}_{2}$ stability curve shown only to establish a baseline with no density stratification. All curves exhibit increasing departures from the incompressible condition. With increasing $\rho_{2} / \rho_{1}$, stability increases (i.e., the amplitude at the most unstable wavelength decreases) and the most unstable wavelength increases, as shown in Fig. $2 \mathrm{~b}$ and listed in Table 5.

Table 2 Mean flow properties, $\rho_{2} / \rho_{1}=1$

\begin{tabular}{lcc}
\hline \hline Mean quantity & $x_{2}=-\infty$ (oxygen) & $x_{2}=\infty$ (oxygen) \\
\hline $\bar{u}_{1}, \mathrm{~m} / \mathrm{s}$ & -193.732 & 193.732 \\
$a_{s}, \mathrm{~m} / \mathrm{s}$ & 484.329 & 484.329 \\
$\bar{\rho}, \mathrm{kg} / \mathrm{m}^{3}$ & 63.191 & 63.191 \\
$p, \mathrm{~atm}$ & 100 & 100 \\
$T, \mathrm{~K}$ & 600 & 600 \\
$Y_{\mathrm{O}}$ & 1 & 1 \\
\hline \hline
\end{tabular}

Table 3 Mean flow properties, $\rho_{2} / \rho_{1}=12.88$

\begin{tabular}{lcc}
\hline \hline Mean quantity & $x_{2}=-\infty$ (oxygen) & $x_{2}=\infty$ (hydrogen) \\
\hline $\bar{u}_{1}, \mathrm{~m} / \mathrm{s}$ & -187.287 & 666.798 \\
$a_{s}, \mathrm{~m} / \mathrm{s}$ & 467.045 & 1671.193 \\
$\bar{\rho}, \mathrm{kg} / \mathrm{m}^{3}$ & 68.271 & 5.298 \\
$p$, atm & 100 & 100 \\
$T, \mathrm{~K}$ & 556 & 444 \\
$Y_{\mathrm{O}}$ & 1 & 0 \\
\hline \hline
\end{tabular}

Table 4 Mean flow properties, $\rho_{2} / \rho_{1}=24.40$

\begin{tabular}{lcc}
\hline \hline Mean quantity & $x_{2}=-\infty$ (oxygen) & $x_{2}=\infty$ (hydrogen) \\
\hline $\bar{u}_{1}, \mathrm{~m} / \mathrm{s}$ & -158.004 & 770.983 \\
$a_{s}, \mathrm{~m} / \mathrm{s}$ & 397.517 & 1915.376 \\
$\bar{\rho}, \mathrm{kg} / \mathrm{m}^{3}$ & 96.764 & 3.965 \\
$p$, atm & 100 & 100 \\
$T, \mathrm{~K}$ & 400 & 600 \\
$Y_{\mathrm{O}}$ & 1 & 0 \\
\hline \hline
\end{tabular}

Table 5 Most unstable wavelength, two-dimensional analysis ${ }^{\mathrm{a}}$

\begin{tabular}{lcccccc}
\hline \hline Case & Flow type & $\rho_{2} / \rho_{1}$ & $\alpha \delta_{\omega}$ & $\alpha \delta_{\omega}\left(c_{i} / \bar{U}\right)$ & $c_{\mathrm{re}} / \bar{U}$ & $\begin{array}{c}\lambda_{1} / \delta_{\omega}= \\
2 \pi / \alpha \delta_{\omega}\end{array}$ \\
\hline 1 & $\begin{array}{c}\text { Variable density } \\
\left(a_{s}=10^{6}\right)\end{array}$ & 1.00 & 0.860 & 0.3830 & 0.6598 & 7.31 \\
& & & & & \\
2 & Variable density $^{\mathrm{c}}$ & 1.00 & 0.797 & 0.3151 & 0 & 7.88 \\
3 & Variable density $^{\mathrm{c}}$ & 12.88 & 0.670 & 0.1756 & -0.0747 & 9.38 \\
4 & Variable density $^{\mathrm{c}}$ & 24.40 & 0.607 & 0.1284 & -0.0745 & 10.35 \\
\hline \hline
\end{tabular}

${ }^{a}$ Here $\alpha$ is a wave number (a real number) and $c$ is a wave speed (a complex number); subscript re denotes real quantity, and subscript $i$ denotes imaginary quantity. $\bar{U}=\Delta U_{0} / 2$.

${ }^{\mathrm{b}}$ Same velocity profile as for case 4 .

${ }^{\mathrm{c}}$ Mean flow in Tables $2-4, M_{c, 0}=0.4$.
Given the computational demands associated with increasing density stratification, all simulations conducted herein will be for the mean flow properties shown in Table 4, and only the Reynolds number $R e_{0}$ will be varied.

Following the arguments of Okong'o and Bellan ${ }^{22}$ showing that three-dimensional eigensolutions to the stability problem are not uniquely defined, the simulations are started with heuristic streamwise and spanwise vorticity perturbations ${ }^{23,24}$ superimposed on the mean initial velocity profile:

$$
\begin{aligned}
& \omega_{1}\left(x_{2}, x_{3}\right)=F_{3 D}\left(\lambda_{1} \Delta U_{0} / \Gamma_{1}\right) f_{2}\left(x_{2}\right) f_{3}\left(x_{3}\right) \\
& \omega_{3}\left(x_{1}, x_{2}\right)=F_{2 D}\left(\lambda_{3} \Delta U_{0} / \Gamma_{3}\right) f_{1}\left(x_{1}\right) f_{2}\left(x_{2}\right)
\end{aligned}
$$

where $\Gamma_{1}$ and $\Gamma_{3}$ are the circulations,

$$
\begin{array}{r}
f_{1}\left(x_{1}\right)=A_{0}\left|\sin \left(\frac{\pi x_{1}}{\lambda_{1}}\right)\right|+A_{1}\left|\sin \left(\frac{\pi x_{1}}{2 \lambda_{1}}\right)\right| \\
+A_{2}\left|\sin \left(\frac{\pi x_{1}}{4 \lambda_{1}}\right)\right|+A_{3}\left|\sin \left(\frac{\pi x_{1}}{8 \lambda_{1}}-\frac{\pi}{2}\right)\right| \\
f_{2}\left(x_{2}\right)=\exp \left[-\pi\left(\frac{x_{2}}{\delta_{\omega, 0}}\right)^{2}\right] \\
f_{3}\left(x_{3}\right)=B_{0} \sin \left(\frac{2 \pi x_{3}}{\lambda_{3}}\right)+B_{1} \sin \left(\frac{\pi x_{3}}{L_{3}}\right)
\end{array}
$$

We use $F_{2 D}=0.1, A_{0}=1, A_{1}=0.5$, and $A_{2}=A_{3}=0.35$ for the streamwise perturbations and $F_{3 D}=0.05, B_{0}=1$, and $B_{1}=0.025$ for the spanwise perturbations. The wavelength of the perturbation is $\lambda_{1}=7.29 \delta_{\omega, 0}$ (the most unstable wavelength for incompressible flow) and $\lambda_{3}=0.6 \lambda_{1}$, following Ref. 23 . The initial vorticity thickness $\delta_{\omega, 0}$ is $6.859 \times 10^{-3} \mathrm{~m}$. The grid is chosen for all simulations to accommodate four wavelengths in the streamwise and spanwise directions, and the evolution of the layer is meant to encompass rollup and two pairings of the initial spanwise vortices. For these initial conditions, the grid sizes and the resolutions are given in Table 6 .

\section{Results}

The results of the simulations listed in Table 6 are discussed. For the first simulation, the value of Reynolds number $R e_{0}$ is chosen to be $6 \times 10^{2}$ to emulate a condition that led to a transitional state for the heptane/nitrogen mixing layer studied by Okong'o and Bellan'; however, that mixing layer had an initial density stratification of 12.88 instead of the much larger value of 24.40 employed in the present study. Unlike the heptane/nitrogen layer at $R e_{0}=6 \times 10^{2}$, the present $\mathrm{O}_{2} / \mathrm{H}_{2}$ layer at the same Reynolds number $R e_{0}$ did not reach transition. The larger $R e_{0}=7.5 \times 10^{2}$ of the second simulation represents an effort to enhance the probability of reaching a transitional state, a strategy that was successful. To understand the physics associated with these results, we first focus on the global characteristics of the layer with special emphasis on the features indicative of transition. Furthermore, we investigate the specific aspects of the instantaneous fields that are inherently absent from the measures given by the global characteristics. Finally, to understand some of the peculiarities of the instantaneous fields, we conduct a second-order statistical analysis focusing on the dissipation.

\section{A. Global Growth Characteristics}

One of the essentialcharacteristics of a mixing layer is its growth. Although many definitions of growth appear in the literature, Cortesi et al. ${ }^{25}$ showed that several such measures, including the momentum thickness, are qualitatively similar. Here, we define the momentum

Table 6 Simulations and associated resolution

\begin{tabular}{lccccccc}
\hline \hline Run & $R e_{0} \times 10^{-2}$ & $L_{1} \times L_{2} \times L_{3}, \mathrm{~m}^{3}$ & $N_{1} \times N_{2} \times N_{3}$ & $R e_{m} \times 10^{-3}$ & $t_{\max }^{*}$ & Time steps & CPU, $\mathrm{h}$ \\
\hline R600 & 6 & $0.2 \times 0.232 \times 0.12$ & $288 \times 336 \times 176$ & 1.195 & 175.32 & 5,150 & 7,132 \\
R750 & 7.5 & $0.2 \times 0.2 \times 0.12$ & $352 \times 352 \times 208$ & 1.680 & 190.44 & 6,860 & 13,214 \\
\hline \hline
\end{tabular}




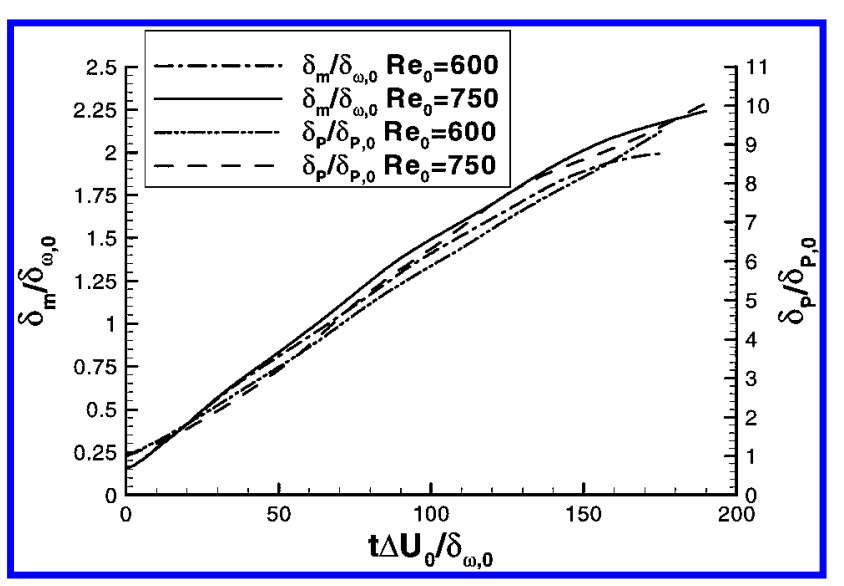

Fig. 3a Momentum thickness and product thickness vs $t^{*}=t \Delta U_{0} / \delta_{\omega, 0}$.

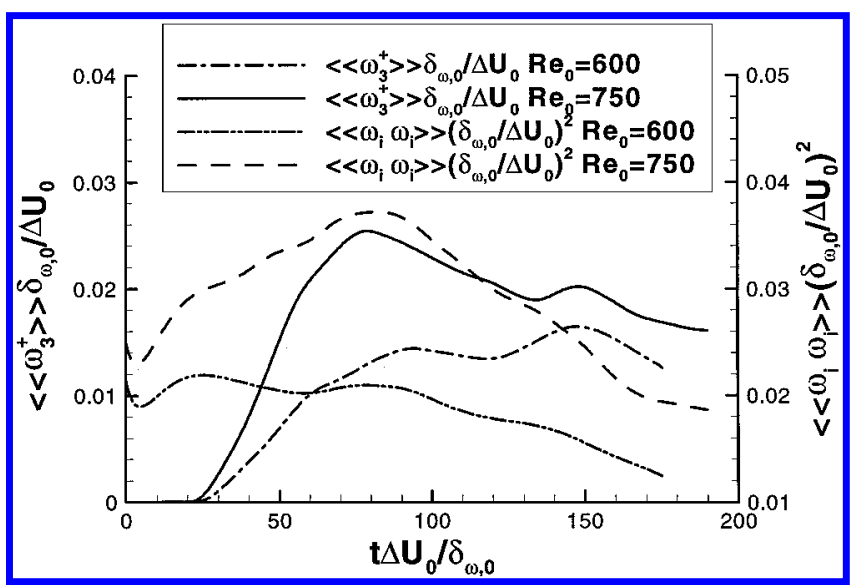

Fig. 3b Global positive spanwise vorticity and enstrophy vs $t^{*}=$ $t \Delta U_{0} / \delta_{\omega, 0}$.

thickness as

$$
\delta_{m}=\frac{-1}{\left(\theta_{1}-\theta_{2}\right)^{2}} \int_{-L_{2, \text { min }}}^{L_{2, \max }}\left(\theta_{2}+\left\langle\rho u_{1}\right\rangle\right)\left(\theta_{1}+\left\langle\rho u_{1}\right\rangle\right) \mathrm{d} x_{2}
$$

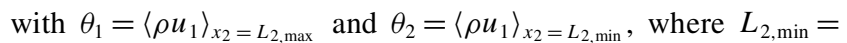
$-L_{2} / 3$ and $L_{2, \max }=2 L_{2} / 3$. Whereas the growth is mostly a consequence of entrainment, the product thickness defined as

$$
\delta_{p}=\iiint_{V} \rho Y_{p} \mathrm{~d} V
$$

in mass units, where $Y_{p}=2 \min \left(Y_{\mathrm{O}}, Y_{\mathrm{H}}\right)$, is a direct consequence of molecular mixing, as also explained by Cortesi et al. ${ }^{25}$ Both of these quantities, nondimensionalized, are illustrated vs the nondimensional time $t^{*}=t \Delta U_{0} / \delta_{\omega, 0}$ in Fig. 3a for the R600 and R750 simulations. (Initial conditions for Figs. $3 \mathrm{a}$ and $3 \mathrm{~b}$ are listed in Tables 4 and 6.) The nondimensional momentum thickness $\delta_{m} / \delta_{\omega, 0}$ of both layers is similar. Both layers exhibit the first pairing at $t^{*}=80$; a second pairing is displayed by the R600 layer at $t^{*}=145$ and by the R750 layer at $t^{*}=150$. Compared to other initially densitystratified layers, such as drop laden layers (cf. Miller and Bellan, ${ }^{26}$ with stratification of 1.5), or to supercritical layers of lesser initial stratification (cf. Okong'o and Bellan, ${ }^{9}$ with stratification of 12.88), the present layers do not show the characteristic $\delta_{m} / \delta_{\omega, 0}$ plateaus indicative of the influence of the forcing. This is attributed to the much larger present stratification, resulting in a resistance to entrainment. The nondimensional product thickness $\delta_{p} / \delta_{p, 0}$ displays a continuous growth indicating that, even for the R600 layer that does not transition, molecular mixing proceeds unabated.

Rotational global features of the layers are shown in Fig. 3b: the nondimensional positive spanwise vorticity $\left\langle\left\langle\omega_{3}^{+}\right\rangle\right\rangle\left(\delta_{\omega, 0} / \Delta U_{0}\right)$ and the nondimensional enstrophy $\left\langle\left\langle\omega_{i} \omega_{i}\right\rangle\right\rangle\left(\delta_{\omega, 0} / \Delta U_{0}\right)^{2}$, where $\boldsymbol{\omega}=\nabla \times \boldsymbol{u}$ is the vorticity. Here $\langle\langle\rangle\rangle$ denotes volume averaging. Because the initial mean velocity profile is such that the initial spanwise vorticity is negative, $\left\langle\left\langle\omega_{3}^{+}\right\rangle\right\rangle\left(\delta_{\omega, 0} / \Delta U_{0}\right)$ is an indicator of the development of small turbulent scales. Complementing this information, $\left\langle\left\langle\omega_{i} \omega_{i}\right\rangle\right\rangle\left(\delta_{\omega, 0} / \Delta U_{0}\right)^{2}$ is a manifestation of stretching and tilting, the mechanism that is primarily responsible for the formation of small scales. For both simulations, $\left\langle\left\langle\omega_{3}^{+}\right\rangle\right\rangle\left(\delta_{\omega, 0} / \Delta U_{0}\right)$ increases from the null value once the layer rollup is completed; however, a reduced augmentationrate is displayed by the R600 simulation corresponding to the reduced layer growth. The first peak in the curves occurs at the first vortex pairing. Another peak is displayed by both layers at the time station of the second pairing; however, this peak constitutes the maximum for the R600 layer, whereas it represents a decay in $\left\langle\left\langle\omega_{3}^{+}\right\rangle\right\rangle\left(\delta_{\omega, 0} / \Delta U_{0}\right)$ for the R750 layer. At the time corresponding to the peak of positive spanwise vorticity, the momentum-thickness-based Reynolds number $R e_{m}$ is $1.122 \times 10^{3}$ for the R600 layer and $1.507 \times 10^{3}$ for the R750 layer. The major difference between the transitional and nontransitional case is shown by the $\left\langle\left\langle\omega_{i} \omega_{i}\right\rangle\right\rangle\left(\delta_{\omega, 0} / \Delta U_{0}\right)^{2}$ plots. For the R600 layer, small scales are initially formed but decay past rollup; in contrast, for the R750 layer the enstrophy culminates, with a substantial peak, at the first vortex pairing and, despite its further decay, remains substantial. Further support of the nontransitional/ransitional aspect of the R600/R750 layer is found in the streamwise and spanwise energy spectra plots (not shown). Although the streamwise spectra displayed a smooth behavior for the two layers, the spanwise spectra for the R600 layer exhibit large jagged peaks, whereas those for the R750 layer are smooth, consistent with the emergence of a continuum of scales indicative of the turbulence regime.

The analysis presented next explores the characteristics of the R750 layer and confirms the global growth results concerning transition.

\section{B. Vorticity Production}

To explore the global rotational state of the R750 layer, we examine the budget of the spanwise vorticity in the $x_{1}-x_{3}$ homogeneous planes and inspect both the average and the rms. A parallel examination is conducted for the vorticity magnitude squared.

The vorticity equation for a compressible flow is

$$
\frac{\mathrm{D} \boldsymbol{\omega}}{\mathrm{D} t}=(\boldsymbol{\omega} \cdot \nabla) \boldsymbol{u}-(\nabla \cdot \boldsymbol{u}) \boldsymbol{\omega}-\nabla\left(\frac{1}{\rho}\right) \times \nabla p+\nabla \times\left(\frac{1}{\rho} \nabla \cdot \overline{\bar{\tau}}\right)
$$

where $\mathrm{D} / \mathrm{D} t$ is the substantialderivative, and the equivalentequation for the vorticity magnitude squared follows:

$$
\begin{aligned}
& \frac{\mathrm{D}(\boldsymbol{\omega} \cdot \boldsymbol{\omega})}{\mathrm{D} t}=2 \boldsymbol{\omega} \cdot(\boldsymbol{\omega} \cdot \nabla) \boldsymbol{u}-2(\nabla \cdot \boldsymbol{u}) \boldsymbol{\omega} \cdot \boldsymbol{\omega}-2 \boldsymbol{\omega} \cdot \nabla\left(\frac{1}{\rho}\right) \\
& \times \nabla p+2 \boldsymbol{\omega} \cdot \nabla \times\left(\frac{1}{\rho} \nabla \cdot \overline{\bar{\tau}}\right)
\end{aligned}
$$

The first term in Eqs. (39) and (40) represents the stretching and tilting contribution, the second term describes the effect of dilatation, the third term is the baroclinic participation to vorticity production, and the last term portrays the viscous contribution. The nondimensional average and $\mathrm{rms}$ of the spanwise vorticity budget at $t^{*}=80$ are shown in Figs. $4 \mathrm{a}$ and $4 \mathrm{~b}$ and correspond to the end of the first pairing. Most of the spanwise rotational activity of the layer, both average and rms, is located in the $\mathrm{H}_{2}$ side, where the lighter fluid is situated. The average spanwise vorticity budget is dominated by large peaks of the viscous term, whereas at some locations the stretching and tilting term competes with the viscous term. On the $\mathrm{O}_{2}$ side of the layer, the activity is dominated by the baroclinic term, whereas the positive dilatation contribution rivals in magnitude the negative stretching and tilting term. Compared to the average spanwise vorticity, the rms displays a large culmination of the stretching and tilting term at the boundary between the two species, indicating that in this crucial region of small-scaleformation there is a considerable activity, explaining the large enstrophy peak at this time station. On the $\mathrm{H}_{2}$ side of the layer, stretching and tilting and viscous terms contribute similarly to the rms and dominate the dilatation and baroclinic term; on the $\mathrm{O}_{2}$ side of the layer, the viscous term dominates, although there is appreciable activity in all other terms. The indication is that, in the $\mathrm{O}_{2}$ side, the formed turbulent scales are dissipated 

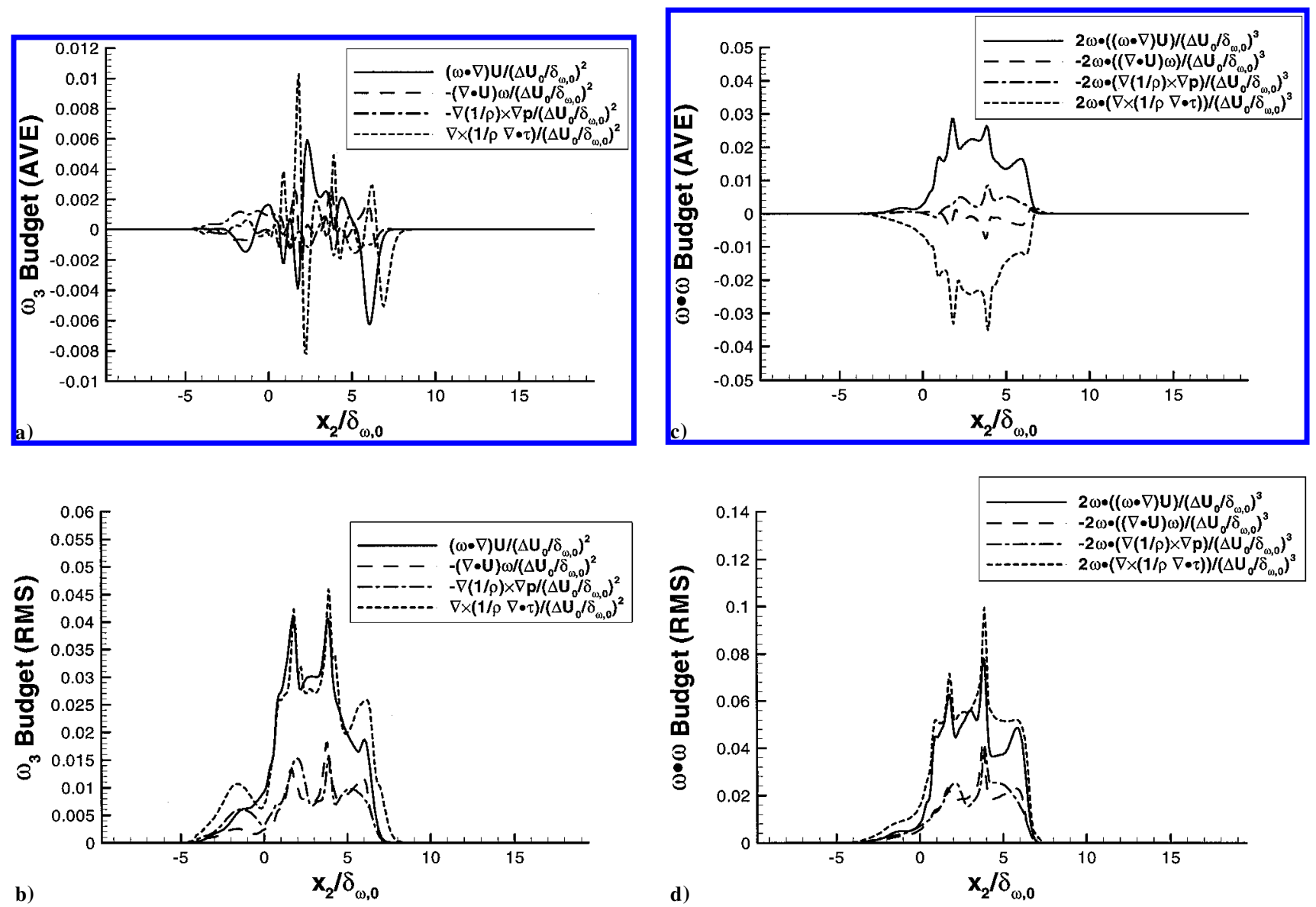

Fig. 4 Run R750 (Table 6) at $t^{*}=80:$ a) plane average of the spanwise vorticity budget, b) plane rms of the spanwise vorticity budget, c) plane average of the vorticity magnitude square budget, and d) plane rms of the vorticity magnitude square budget.

by the action of viscosity. The result of this dissipation is clearly seen in Figs. 4c and 4d, showing the nondimensional average and rms of the vorticity magnitude squared budget. The viscous term is larger in magnitude than all other terms and negative, whereas the second term in order of decreasing magnitude is the stretching and tilting term, which is positive, thus, indicating production of vorticity by this mechanism. Production through all mechanisms is negligible in the $\mathrm{O}_{2}$ side of the layer, and the insignificant amount produced is dissipated by the dominating viscous effect. Finally, the rms of the vorticity magnitude budget shows the same ordering of terms as the rms of the spanwise vorticity budget.

To understand the timewise evolution of the layer, Figs. 5a and $5 \mathrm{~b}$ show the nondimensional average and rms of the spanwise vorticity budget at $t^{*}=150$. Similar to the $t^{*}=80$ situation, most of the spanwise rotational activity of the layer, both average and rms, is located on the $\mathrm{H}_{2}$ side. Compared to the magnitude of the equivalent terms at $t^{*}=80$, all terms are here reduced approximately by a factor of 2 , indicating that vorticity production is abated; this finding is consistent with the global characteristics presented earlier. The dominant contribution to the average spanwise vorticity is from the stretching and tilting term, which is negative, although at some cross-stream locations situated well into the $\mathrm{H}_{2}$ side of the layer, the viscous term rivals the stretching/tilting one in magnitude and is positive. Both the averaged dilatation and baroclinic terms appear much smaller. This ordering of the relative magnitudes is even more dramatic in the rms budget. Clearly, the rms production is primarily due to stretching/tilting and viscosity effects, which are essentially of similar magnitude; dilatation and baroclinic influences are smaller by approximately a factor of 4 . In Figs. 5c and $5 \mathrm{~d}$, a similar evaluation is presented for the vorticity magnitude squared. Although generally the same ordering of stretching/tilting and viscosity vs dilatation and baroclinic terms holds, viscous effects dominate the stretching/tilting activity, and the average viscous contribution seems to extend farther into the $\mathrm{O}_{2}$ side of the layer. As expected, stretching/tilting is responsible for increasing the magnitude of the vorticity squared due to production of small scales, whereas the viscous term diminishes the magnitude of the vorticity through dissipation. The physical picture emerging is that production of small scales does not keep up with dissipation; however, a strong dissipation is possible only because of a previous vigorous production of small scales.

\section{Visualizations of the Dynamic and Thermodynamic Variables}

Instantaneous aspects of the flow may reveal information that is unavailable from global characteristics. Such instantaneous aspects are best illustrated through contour plots at specific times and locations, chosen to highlight important features of the layer. Because two important times are $t^{*}=80$ and 150 , as explained earlier, the flow visualizations will depict the variables at one or both of those times.

\section{Dynamic Variables}

As discussed earlier, one of the most fundamental variables indicating the evolution of the flow is the spanwise vorticity. Shown in Figs. $6 \mathrm{a}$ and $6 \mathrm{~b}$ are braid cross $\operatorname{section}\left(x_{3}=L_{3} / 16=0.0075 \mathrm{~m}\right)$ distributions of the spanwise vorticity at $t^{*}=80$ and 150 , respectively. The between-the-braid plane cross sections $\left(x_{3}=L_{3} / 2=0.06 \mathrm{~m}\right)$ display an equivalent behavior. At $t^{*}=80$, the two vortices remaining after the second pairing are clearly shown, whereas at $t^{*}=150$, only a single vortex appears; however, some remnants of the second pairing process are obvious. The level of the positive spanwise vorticity, indicative of small-scale formation decreases from $t^{*}=80$ to 150 , consistent with the global peak in positive spanwise vorticity as a function of time; however, the maximum positive spanwise vorticity increases in the between-the-braid plane as the layer evolves from the first to the second pairing.

The evolution of the streamwise vorticity in the midbraid plane was documented by many investigators for gases at atmospheric 

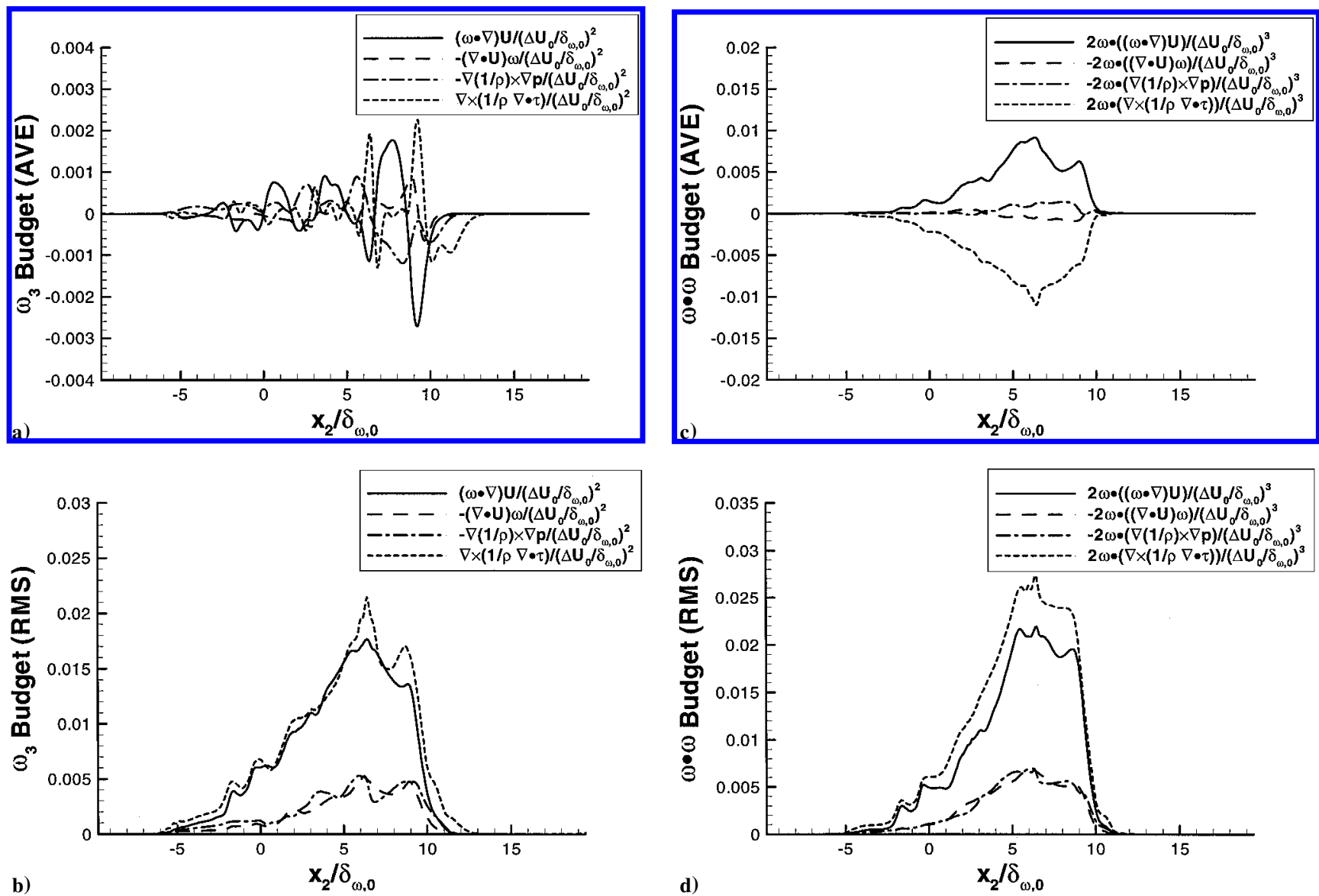

Fig. 5 Run R750 (Table 6) at $t^{*}=150$ : a) plane average of the spanwise vorticity budget, b) plane rms of the spanwise vorticity budget, c) plane average of the vorticity magnitude square budget, and d) plane rms of the vorticity magnitude square budget.

conditions (e.g., Rogers and Moser ${ }^{27}$ ). Of particularinterestfor qualitative comparison with the present results are the plots of Cortesi et $\mathrm{al}^{28}$ for gravitationally density-stratified temporal mixing layers; for such layers, the influence of gravity is measured by the value of the Richardson number. Presented in Figs. 7a and 7b are the present streamwise midbraid plane $\left(x_{1} / \delta_{\omega, 0}=25.6\right)$ contours at $t^{*}=80$ and 150 , respectively. These instantaneous plots may be compared with Figs. 9a and $9 \mathrm{~b}$ of Rogers and Moser, ${ }^{27}$ corresponding to time stations after the first and second pairings, respectively. In both Figs. 7a and 7b, distortions are observed in the cross-stream direction when comparing with the equivalent figures in Ref. 27, which display a definite symmetry. This symmetry of the structures both in the cross-streamand the spanwise directions no longer exists here due to the initial density stratification of the layer. The overwhelming activity is in the $\mathrm{H}_{2}$ side of the layer, containing the lighter fluid. On the other hand, comparisons with Fig. 16a of Cortesi et al. ${ }^{28}$ show that their streamwise vorticity also lacks symmetry; however, those calculations are not quantitatively comparable with ours because of the different forcing, among other different aspects. For example, Cortesi et al. ${ }^{28}$ find that, as the Richardson number is increased, the structures recover some symmetry (their Fig. 17a).

\section{Thermodynamic Variables}

In previous studies of supercritical mixing layers ${ }^{8,9,22}$ for heptane/nitrogen at $60 \mathrm{~atm}$ and freestream temperatures of $T_{1}=1000 \mathrm{~K}$ and $T_{2}=600 \mathrm{~K}$, the peculiarities of the layer were associated with departures from perfect gas behavior $(Z=1$ for a perfect gas) and departures from mixture ideality (for an ideal mixture $\alpha_{D}=1$ ). These associations were issued from quantitative as well as visual correlations. Contour plots of $Z$ and $\alpha_{D}$ for the present $\mathrm{O}_{2} / \mathrm{H}_{2}$ layer (freestream conditions of $100 \mathrm{~atm}, T_{1}=600 \mathrm{~K}$, and $T_{2}=400 \mathrm{~K}$ ) at differenttime stations, both in the braid and in the between-the-braid planes, reveal that the fluid is extremely close to a perfect gas and that the mixture is nearly ideal. For example, at $t^{*}=150$ the compression factor varies between 1.009 and 1.035 , whereas the mass diffusion factor varies between 0.9939 and 0.9996 . Therefore, none of the features to be discussed can be associated with specific real gas or nonideal mixture behavior. However, note that this thermodynamic state of the layer could not be foreseen a priori, and it is only a result of the calculation. (Moreover, it is expected that at the same pressure, with decreasing temperaturesthe mixture will increasingly exhibit departures from perfect gas and ideal mixture conditions.)

One of the most distinctive features of supercriticalmixing layers examined so far is the existence of regions of high density-gradientmagnitude (HDGM). These regions have been identified in both pretransitiona $^{\beta, 22}$ and transitional ${ }^{\uparrow, 22}$ supercritical mixing layers. Given the perfect gas and near ideal conditions of this layer, in retrospect these distinctive features could perhaps be better associated with the initial density stratification. However, movie animations of the $|\nabla \rho|$ timewise evolution show that the origin of these regions is not only from the distortion of the initial boundary between the two fluid species, but also from the mixing between the two species; this conclusion holds for all supercritical mixing layers studied so far, independent of the binary system of species. Figs. 8a and 8b show $|\nabla \rho|$ in the braid and the between-the-braid cross sections located at $x_{3}=0.0075$ and $0.06 \mathrm{~m}$, respectively, at $t^{*}=150$. Compared to heptane/nitrogen mixing layers excited at the same wavelength and $F_{3 D}$ (Ref. 9), the present regions of high $|\nabla \rho|$ are more spread out, particularlyin the between-the-braidplane. The increased spreading of the HDGM structures is attributed to the increased solubility in the $\mathrm{O}_{2} / \mathrm{H}_{2}$ system with respect to the heptane/nitrogen one. For example, in studies of heptane drops in nitrogen, Harstad and Bellan ${ }^{15}$ found that the initial density discontinuity is maintained, although the location of the discontinuity changes during the drop evolution. In contrast, a $\mathrm{O}_{2}$ drop in $\mathrm{H}_{2}$ displays a quicker relaxation of $Y_{\mathrm{O}}$ from unity inside the drop, indicating important $\mathrm{H}_{2}$ solubility effects. ${ }^{18}$

Because the existence of the HDGM regions cannot be here associated with real gas effects, or entirely due to the initial density stratification, the question arises as to their origin. Clearly, they 

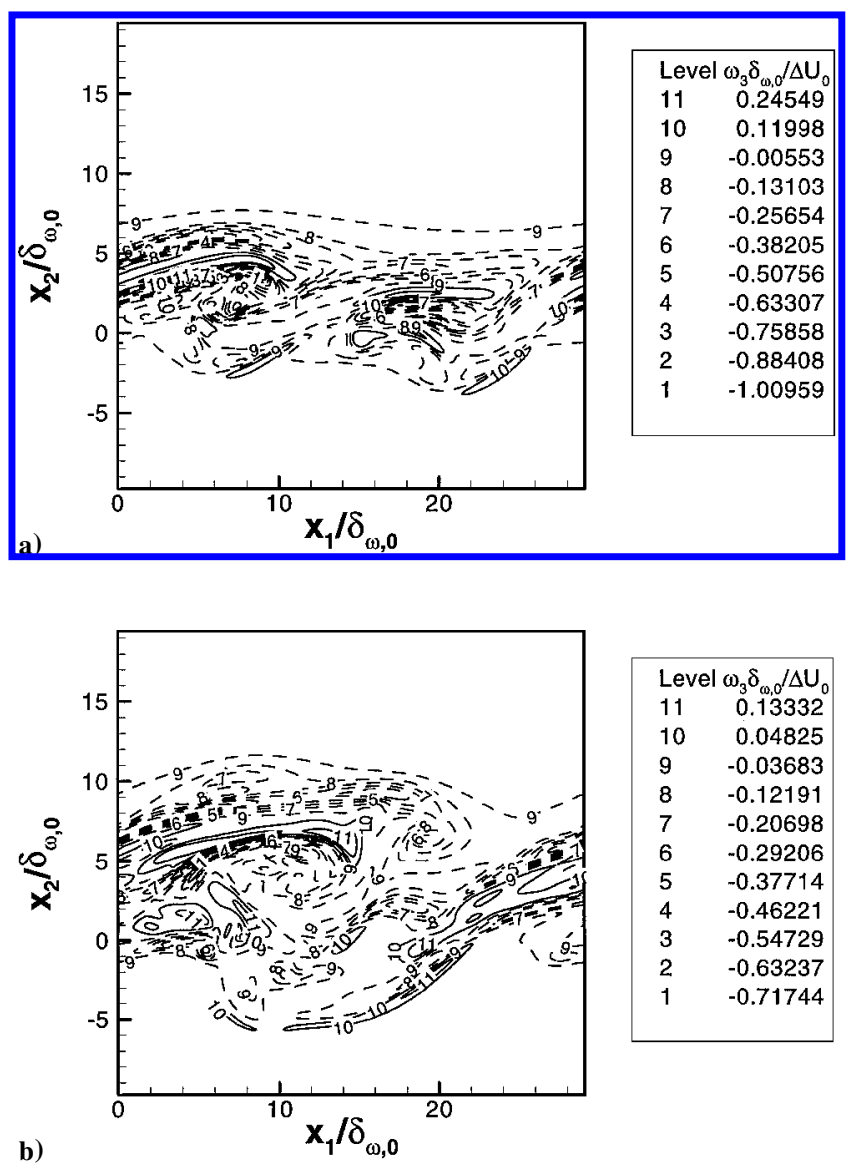

Fig. 6 Run R750 (Table 6) nondimensional spanwise vorticity in the a) braid plane $\left(x_{3}=0.0075 \mathrm{~m}\right)$ at $t^{*}=80$ and $\left.\mathrm{b}\right)$ braid plane at $t^{*}=150$.
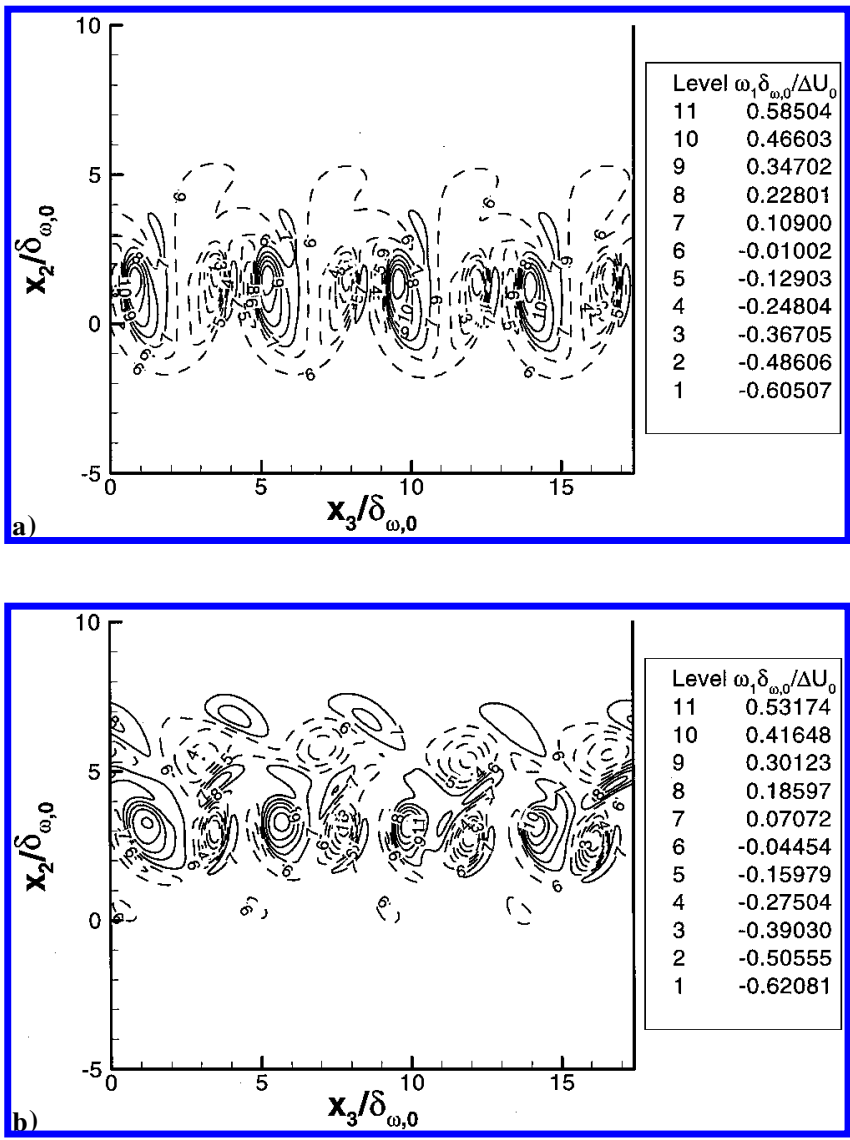

Fig. 7 Run R750 (Table 6) nondimensional streamwise vorticity in the a) streamwise midbraid plane $\left(x_{1} / \delta_{\omega, 0}=25.6\right)$ at $t^{*}=80$ and b) midbraid plane at $t^{*}=150$.
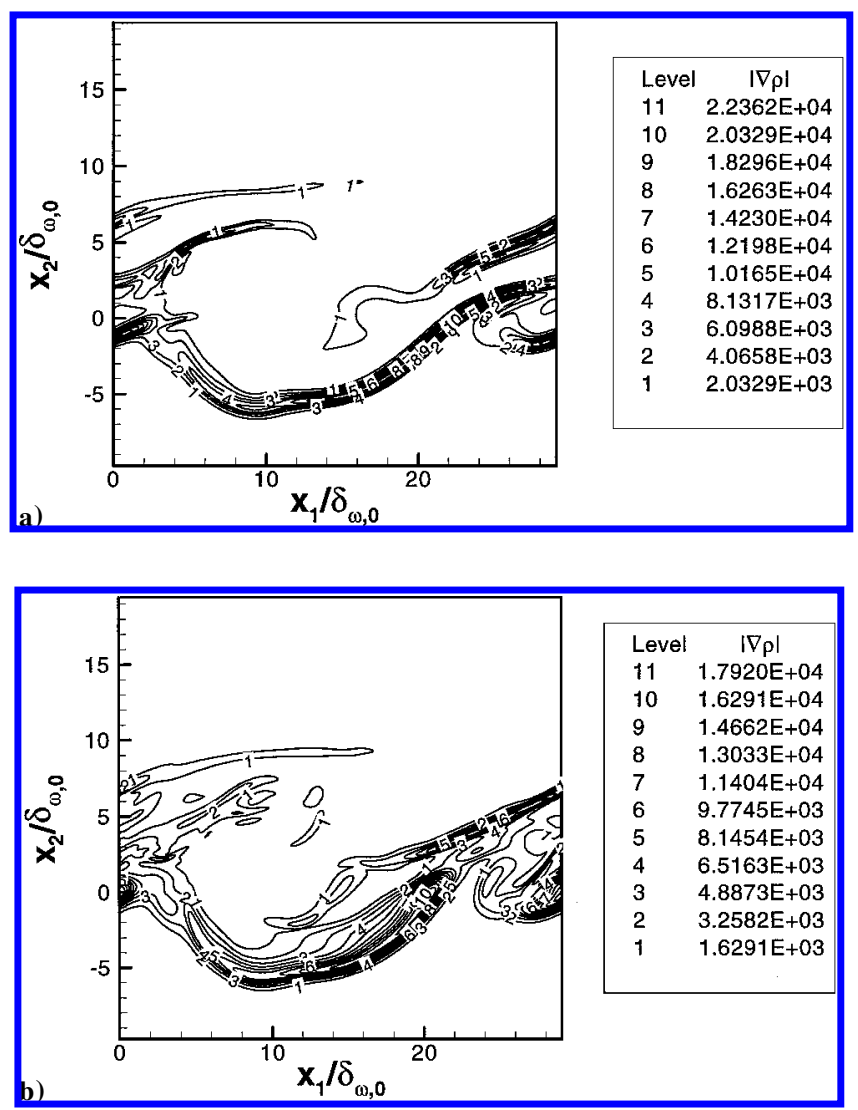

Fig. 8 Run R750 (Table 6) at $t^{*}=150$, density gradient magnitude in the a) braid plane $\left(x_{3}=0.0075 \mathrm{~m}\right)$ and $\left.\mathrm{b}\right)$ between-the-braid plane $\left(x_{3}=\right.$ $0.06 \mathrm{~m}$ ) (dimension is $\left.\mathrm{kg} / \mathrm{m}^{4}\right)$.

are the result of mixing, without which they would not be formed. Parcels of heavy $\mathrm{O}_{2}$ are entrained into the lighter $\mathrm{H}_{2}$, and they lose their identity only after mixing at the small scale. Before that time, the much larger molar weight of $\mathrm{O}_{2}$ gives rise to a substantialdensity gradient. Therefore, the HDGM regions are here attributed to the very large molar weight ratio (a factor of 16) between $\mathrm{O}_{2}$ and $\mathrm{H}_{2}$. Contrary to the situation encountered for heptane/nitrogen, where diffusion (which is a molecular scale process) was inhibited by the lower than unity mass diffusion factor, here such diffusion is efficient $\left(\alpha_{D} \sim 1\right)$. Thus, we find that, generally, HDGM regions may occur under quite different circumstances. For large molar weight ratio species, these regions may occur even if the species diffuse well. (Note again that this is a molecular scale process.) For species having a modest to low molar weight ratio, HDGM regions may still occur if the species have difficulty in diffusing into each other.

To investigate the visual correlation between the HDGM regions and the distribution of $Y_{\mathrm{O}}$, contour plots of the $\mathrm{O}_{2}$ mass fraction at $t^{*}=150$ are shown in Figs. 9a and $9 \mathrm{~b}$ representing the braid and the between-the-braid planes. As inferred earlier, some parcels of $\mathrm{O}_{2}$ have broken off from the lower stream and are seen convected to the upper stream. Also, the layer consists of substantially inhomogeneous fluid composed of both $\mathrm{O}_{2}$ and $\mathrm{H}_{2}$. An equivalent physical picture of nonhomogeneities is obtained when examining the $Y_{\mathrm{O}}$ distribution at $t^{*}=150$ in the streamwise midbraid plane located at $x_{1} / \delta_{\omega, 0}=25.6$ (Fig. 9c). The characteristic mushroom shapes typical of the streamwise plane of three-dimensionalsimulations are evident; equivalent $|\nabla \rho|$ contours (not shown) are found. The disintegration of the lower $\mathrm{O}_{2}$ stream and the migration of parcels of $\mathrm{O}_{2}$ into the $\mathrm{H}_{2}$ stream are manifest. Noteworthy, Cortesi et al. ${ }^{25}$ also detected these mushroom structures (their Fig. 9a) evolving during the simulation of gravitationallydensity-stratified three-dimensionalmixing layers forced deterministically.These structureswere less developed when a combined deterministic-randomvelocity field was used during the initialization.

To quantitatively assess the composition of the HDGM regions, averages of $Y_{\mathrm{O}}$ conditioned on percentages of $|\nabla \rho|$ are listed in 

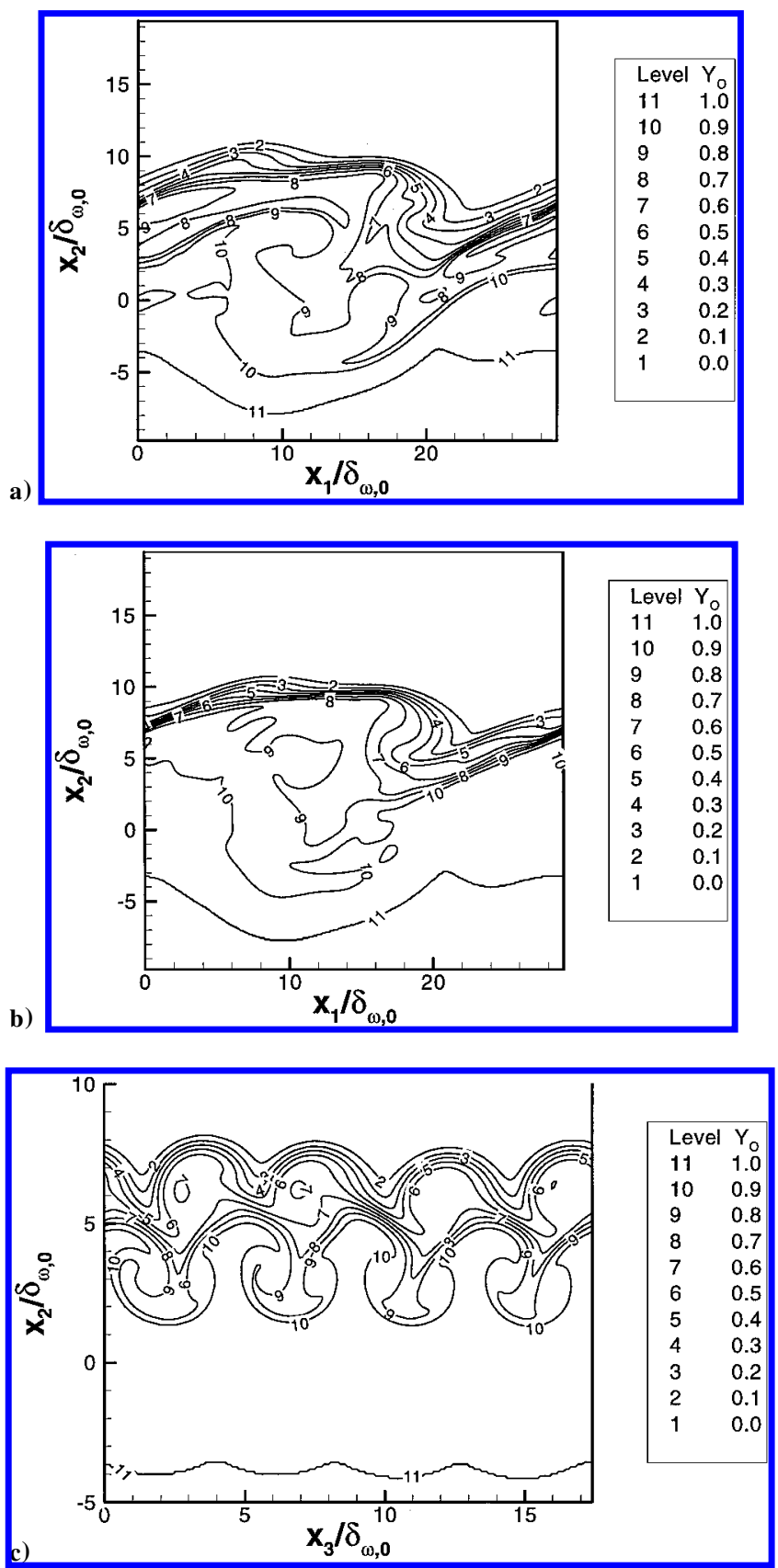

Fig. 9 Run R750 (Table 6), $t^{*}=150$, oxygen mass fraction in the a) braid plane $\left(x_{3}=0.0075 \mathrm{~m}\right)$, b) between-the-braid plane $\left(x_{3}=0.06 \mathrm{~m}\right)$, and c) streamwise midbraid plane $\left(x_{1} / \delta_{\omega, 0}=25.6\right)$.

Table 7 representing results at $t^{*}=150$; a similar calculation performed at $t^{*}=80$ yielded numbers similar up to the third digit. The conditional averages are calculated over regions of $|\nabla \rho|>$ $|\nabla \rho|_{\text {cutoff }} \equiv K|\nabla \rho|_{\max }$ with $K=0.1,0.2,0.3$, and 0.4. As higher values of $|\nabla \rho|$ are probed, the mass fraction of $\mathrm{O}_{2}$ increases, similarly to the findings with the heptane/nitrogen system ${ }^{9}$; however, for the same $K$, the average mass fraction values are here higher. When it is considered that the stoichiometric $\mathrm{O}_{2}$ mass fraction is here $Y_{\mathrm{O}, s}=32 /(32+4)=0.89$, being calculated from the reaction

$$
2 \mathrm{H}_{2}+\mathrm{O}_{2} \rightarrow 2 \mathrm{H}_{2} \mathrm{O}
$$

the present values of $0.906-0.956$ found from conditional averages seem to indicate a globally favorable situation for combustion purposes. In contrast, for heptane/air the stoichiometric heptane mass fraction calculated from the reaction

$\mathrm{C}_{7} \mathrm{H}_{16}+11 \mathrm{O}_{2}+(3.7 \times 11) \mathrm{N}_{2} \rightarrow 7 \mathrm{CO}_{2}+8 \mathrm{H}_{2} \mathrm{O}+(3.7 \times 11) \mathrm{N}_{2}$
Table 7 Conditional averages of $Y_{O}$ over regions where $|\nabla \rho|>|\nabla \rho|_{\text {cutoff }}{ }^{a}$

\begin{tabular}{lcc}
\hline \hline $\begin{array}{l}|\nabla \rho|_{\text {cutoff }}= \\
K|\nabla \rho|_{\text {max }}\end{array}$ & $\begin{array}{c}\text { Braid plane, } \\
|\nabla \rho|_{\max }= \\
2.440 \times 10^{4} \mathrm{~kg} / \mathrm{m}^{4}\end{array}$ & $\begin{array}{c}\text { Between-the-braid } \\
\text { plane, }|\nabla \rho|_{\text {max }}= \\
1.955 \times 10^{4} \mathrm{~kg} / \mathrm{m}^{4}\end{array}$ \\
\hline$K=0.4$ & 0.954 & 0.956 \\
$K=0.3$ & 0.945 & 0.951 \\
$K=0.2$ & 0.930 & 0.949 \\
$K=0.1$ & 0.906 & 0.923 \\
\hline \hline
\end{tabular}

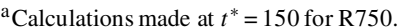

\begin{tabular}{lc} 
Table 8 & $\begin{array}{c}\text { Correlations with }|\nabla \rho| \text { at } \\
\boldsymbol{t}^{*}=\mathbf{1 5 0} \text { for R750 }\end{array}$ \\
\hline \hline Region & $Y_{\mathrm{O}},|\nabla \rho|$ correlation \\
\hline Braid plane & 0.34 \\
Between-the-braid plane & 0.37 \\
Volume-based & 0.35 \\
\hline \hline
\end{tabular}

is $Y_{h, s}=100 /(11 \times 32+3.7 \times 11 \times 28)=0.067$, which compares less favorably with values of $0.84-0.921$ found from similar conditional averages with $K=0.1,0.2$, and 0.3 using the results of a heptane/nitrogen simulation. ${ }^{9}$ This comparison is only qualitatively correct because the simulations in Ref. 9 were based on the heptane/nitrogen instead of the heptane/air system.

To further assess the correlation between $|\nabla \rho|$ and $Y_{\mathrm{O}}$, Table 8 is a list of the coefficients found in the braid and the between-thebraid planes at $t^{*}=150$. The volume-based correlation between two variables is defined by

$$
\mathcal{R}_{2}(\mathcal{X}, \mathcal{Y})=\frac{\langle\langle\mathcal{X} \mathcal{Y}\rangle\rangle-\langle\langle\mathcal{X}\rangle\rangle\langle\langle\mathcal{Y}\rangle\rangle}{\sqrt{\left(\left\langle\left\langle\mathcal{X}^{2}\right\rangle\right\rangle-\langle\langle\mathcal{X}\rangle\rangle^{2}\right)\left(\left\langle\left\langle\mathcal{Y}^{2}\right\rangle\right\rangle-\langle\langle\mathcal{Y}\rangle\rangle^{2}\right)}}
$$

where $\mathcal{X}$ and $\mathcal{Y}$ are generic variables. The correlation in Table 8 is moderate and similar to that found in a previous study for heptane/nitrogen.

Because the temperature is directly related to the density through the EOS, the expectation is that the temperature distribution will be visually highly correlated with $|\nabla \rho|$ if the pressure is approximately constant. Indeed, examination of pressure contours in both the braid and the between-the-braidplanes (not shown) reveals that the variations from the initial uniform pressure are small, at most $8 \%$. Consistent with the almost uniform pressure, the braid and the between-the-braid temperature contours (not shown) are visually well correlated with $|\nabla \rho|$. Hotter fluid from the upper stream is transported to the lower stream, and the HDGM regions generally contain fluid at higher temperature than their surroundings. This situation is very beneficial to combustion because it has been already determined that in these regions the composition is close to stoichiometric.

\section{Irreversible Entropy Production (Dissipation)}

The dissipation, which is the irreversible entropy production, is a quantity intimately associated with turbulence. If $g$ denotes the rate of irreversible entropy production, then according to Okong'o and Bellan $^{10}$

$$
\begin{gathered}
g=g_{\text {visc }}+g_{\text {temp }}+g_{\text {mass }} \\
g_{\text {visc }}=\frac{\mu}{T}\left(2 S_{i j} S_{i j}-\frac{2}{3} S_{k k} S_{l l}\right), \quad g_{\text {temp }}=\frac{\lambda}{T^{2}} \frac{\partial T}{\partial x_{j}} \frac{\partial T}{\partial x_{j}} \\
g_{\text {mass }}=\frac{1}{Y_{\mathrm{H}} Y_{\mathrm{O}} \rho D} \frac{R_{u} m}{m_{\mathrm{H}} m_{\mathrm{O}}} j_{\mathrm{O} j} j_{\mathrm{O} j} \\
S_{i j}=\frac{1}{2}\left(\frac{\partial u_{i}}{\partial x_{j}}+\frac{\partial u_{j}}{\partial x_{i}}\right)
\end{gathered}
$$

where, according to Eqs. (6-8), $g_{\text {mass }}$ contains the mass diffusion factor $\left(\propto \alpha_{D}\right)$ and the Soret $\left(\propto \alpha_{\mathrm{BK}}\right)$ terms through $j_{\mathrm{O} j}$. Figures 10a and $10 \mathrm{~b}$ show the braid and the between-the-braid plane distributions of $g$ at $t^{*}=150$, respectively. A visual comparison with the 

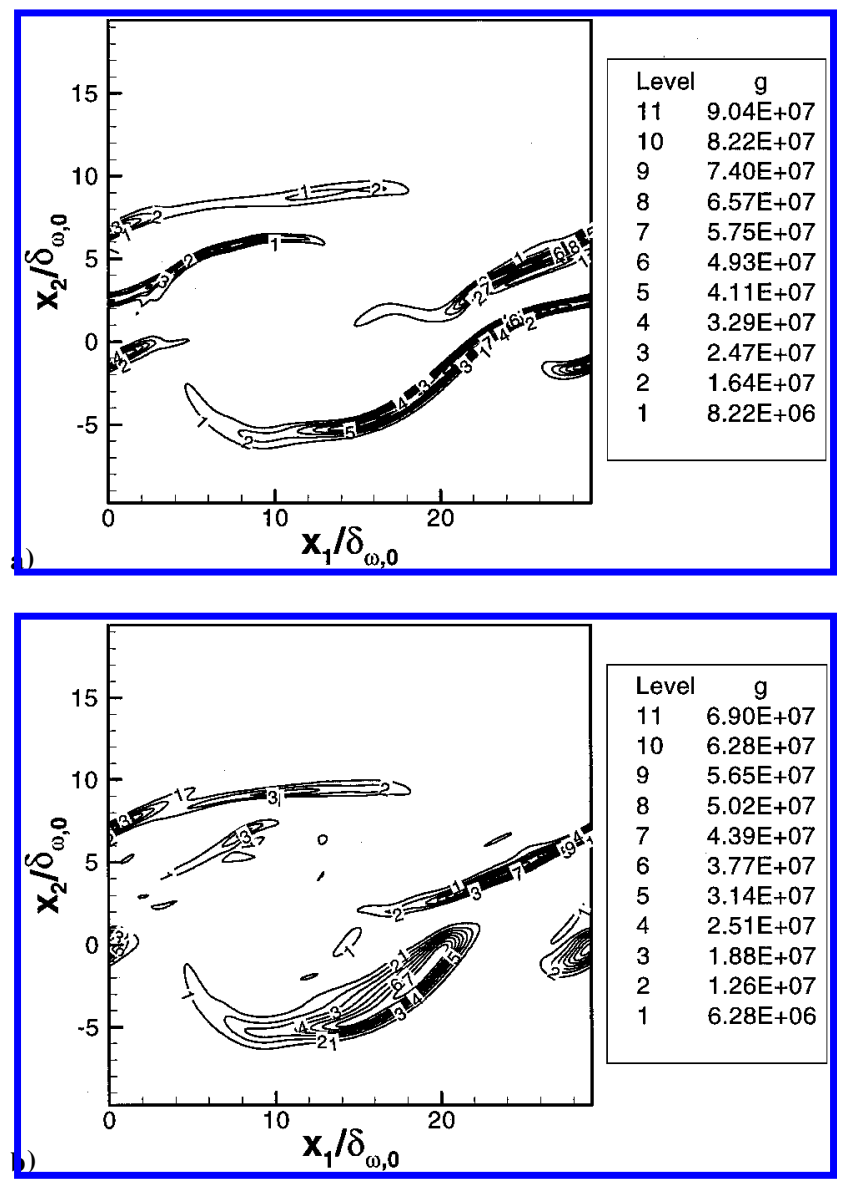

Fig. 10 Run R750 (Table 6), $t^{*}=150$, dissipation in the a) braid plane $\left(x_{3}=0.0075 \mathrm{~m}\right)$ and $\left.\mathrm{b}\right)$ between-the-braid plane $\left(x_{3}=0.06 \mathrm{~m}\right)$ [dimension is $\left.\mathbf{J} /\left(\mathbf{m}^{3} \mathbf{K}\right)\right]$.

$|\nabla \rho|$ plots of Figs. 8a and $8 \mathrm{~b}$ leads to the conclusion that there is a strong correlation between these two quantities; the calculated global correlation is 0.85 , and the braid correlation is 0.86 . These results regarding strong dissipation at a density gradient interface agree with the observations of Hannoun et al., ${ }^{29}$ who experimentally investigated the turbulence structure near a sharp density interface and found that small scales were damped by the region of large $|\nabla \rho|$ that acted similar to a material interface.

To determine the mechanism(s) primarily responsible for the dissipation, homogeneous-plane average plots of the $g_{\text {visc }}, g_{\text {mass }}$, and $g_{\text {temp }}$ contributions to $g$ are shown in Figs. 11a (average) and $11 \mathrm{~b}$ $(\mathrm{rms})$ at $t^{*}=150$; these are typical of the situation at $t^{*}=80$ as well. The contribution from $g_{\text {mass }}$ is a factor of 6 larger than those from $g_{\text {visc }}$ and $g_{\text {temp }}$ for both the average and the rms. The largest peaks in the average (Fig. 11a) are on the $\mathrm{O}_{2}$ side, corresponding to the HDGM region, and at the boundary of the two species, corresponding to large gradients in the mass fractions; the secondary peaks on the $\mathrm{H}_{2}$ region also correspond to the HDGM region. The major peak in the rms (Fig. 11b) corresponds to the boundary between species, with secondary peaks on either side corresponding to the HDGM regions. Most of $g_{\text {visc }}$ is concentrated on the $\mathrm{H}_{2}$ side, where most of the small scales have been created (see Fig. 5). Note that $g_{\text {temp }}$ has a small peak at the boundary between the two species, which also corresponds to the location of the initial temperature stratification. The much smaller $g_{\text {temp }}$ is the result of the enhanced $L e_{\text {eff }}$ at supercritical conditions, as shown by Harstad and Bellan. ${ }^{12}$ The larger Lewis number $L e_{\text {eff }}$ causes the temperature to relax faster than the mass fraction (nondimensionalgradients are smaller), as the larger (than at low-pressure conditions) thermal conductivity promotes heat transfer. It is also apparent that $g_{\text {temp }}$ produces slightly more rms than $g_{\text {visc }}$.

Based on this analysis, it is apparent that supercritical mixing layers are quite different from their atmospheric condition, subcritical counterparts. In these latter mixing layers, the dissipation is
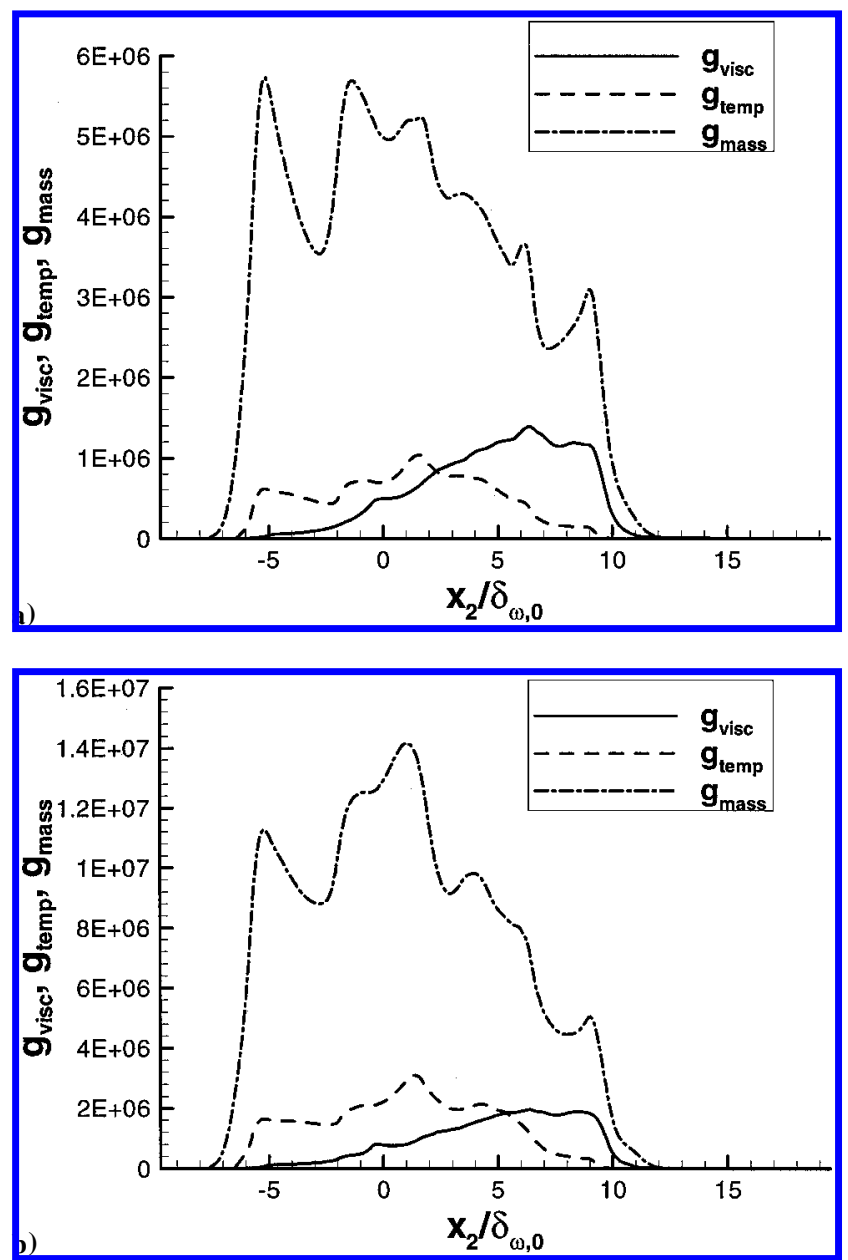

Fig. 11 Run R750 (Table 6), $t^{*}=150$, contributions to the dissipation from viscous, heat flux, and molar flux effects in homogeneous planes: a) average and b) $\mathbf{r m s}$ [dimension is $\left.J /\left(\mathbf{m}^{3} \mathbf{K}\right)\right]$.

mainly of viscous origin, whereas in the supercritical mixing layer, the dissipation is primarily due to mass flux effects. Because it is the dissipation that must ultimately be duplicated by turbulence models, the implication of this finding is that those models focusing on viscosity effects might be misguided when trying to portray supercritical turbulent mixing.

\section{Conclusions}

A DNS study has been performed of a supercritical threedimensional temporal $\mathrm{O}_{2} / \mathrm{H}_{2}$ mixing layer to explore aspects of mixing under shearing conditions. The conservation equations were based on fluctuation-dissipation theory, having an enlarged transport matrix that includes Soret and Dufour effects. To close the system of equations, a real gas EOS was coupled to the differential equations. The Schmidt and Prandtl numbers were accurate as much as possible in the chosen $(p, T)$ regime. This was accomplished by correlating the Schmidt and Prandtl numbers as functions of the thermodynamic variables, consistent with contour plots of these numbers based on accurate properties. The values of these numbers determined the thermal conductivity and diffusivity, whereas the viscosity was determined from the prescribed initial value of the Reynolds number. Boundary conditions were of periodic type in the streamwise and spanwise directions and of nonreflecting outflow type, based on a real gas characteristic analysis of the differential equations, in the cross-stream direction. Additional to the Reynolds number, the initial conditions prescribed the Mach number, the temperatures of the two freestreams, the pressure, and the perturbation of the layer.

Simple calculations showed that, at high pressure, as the oxygen is at lower temperatures, the stratification increases dramatically. A stability analysis conducted for the $\mathrm{O}_{2} / \mathrm{H}_{2}$ system resulted in curves illustrating an increased stability with respect to the incompressible 
case and a most unstable wavelength increasing with increasing stratification.

Based on the stability analysis and previous experience with heptane/nitrogen supercritical mixing layers, the perturbation wavelength was chosen to be the most unstable incompressibleone, and the amplitudes of the excitation were those that previously lead to transition in a similar heptane/nitrogen mixing layer simulation having albeit a smaller initial density stratification. The domain size was four times the perturbation wavelength to accommodate four vortices and two pairings. Two mixing layer conditions were simulated, differing only by the initial Reynolds number. Whereas the smaller initial Reynolds number simulation did not reach transition, the larger initial Reynolds number reached a transitional state. This conclusion was based on consideration of global layer evolution, as well as energy spectra at the transitional state.

For the larger-initial-Reynolds-number layer, global characteristics of the layer showed a momentum thickness and product thickness continuous growth, with a relatively large momentumthickness-based Reynolds number reached. The evolution of the global positive spanwise vorticity and the enstrophy displayed a large peak following the first pairing and continued to decay afterwards with only a minor increase following the second pairing.

To understand the rotational characteristics of the layer indicated by the global evolution, the vorticity and vorticity magnitude budgets were scrutinized at times following each pairing. Consistent with the global growth characteristics, very little vorticity is created on the oxygen side of the layer, which contains the heavier fluid. Most of the vorticity is created on the hydrogen side of the layer by the action of the stretching/tilting term. Even in those regions, the negative viscous term dominates the budget of the vorticity magnitude squared, draining vorticity from the system and explaining the eventual decrease in the global positive spanwise vorticity.

Visualizations of the dynamic and thermodynamic variables revealed regions of HDGM, which mostly exist in the lower $\mathrm{O}_{2}$ stream. These regions are the result of both the distortion of the initial density stratification boundary and the mixing of the two fluids. Because of the very large molar weight ratio between oxygen and hydrogen, parcels of $\mathrm{O}_{2}$ detached from the lower stream maintain their density identity while being entrained into the upper, lighter hydrogen, thereby creating these large density-gradient-magnitude regions before the complete mixing of the two fluids. Although regions of HDGM also were identified during the mixing of heptane/nitrogen, in that situation they were quantitatively correlated with locations of mixture nonideality. In contrast, at the conditions of the present simulations, the fluid behaves as a perfect gas and an ideal mixture.

At the transitional state, inspection of the irreversible entropy production, which is the dissipation, revealed a strong correlation with the HDGM regions. In contrast to atmospheric mixing layers where most of the dissipation is of viscous origin, here it was found that the overwhelming contribution is from the species mass flux, making the two situations fundamentally different. This result indicates that turbulence models focusing on viscosity effects might not be portraying the correct physics under supercritical conditions.

These findings are the result of examining a single realization, and additional studies at different temperatures are necessary to generalize these preliminary conclusions. Simulations at lower temperatures, where real gas and mixture nonideality effects are expected to play an important role, will be more relevant to $\mathrm{O}_{2}$ disintegration.

\section{Appendix: Mixing Rules}

Miscellaneous relationships relevant to the EOS are

$$
a_{m}=\sum_{i} \sum_{j} X_{i} X_{j} a_{i j}(T), \quad b_{m}=\sum_{i} X_{i} b_{i}
$$

where indices here do not follow the Einstein notation, and

$$
\begin{gathered}
a_{i j}=0.457236\left[\left(R_{u} T_{c, i j}\right)^{2} / p_{c, i j}\right] \alpha_{i} \alpha_{j} \\
\alpha_{i} \equiv 1+C_{i}-C_{i} \sqrt{T / T_{c, i}} \\
C_{i}=0.37464+1.54226 \Omega_{i}-0.26992 \Omega_{i}^{2} \\
b_{i}=0.077796\left(R_{u} T_{c, i} / p_{c, i}\right)
\end{gathered}
$$

$$
\begin{gathered}
T_{c, i j}=\left(1-k_{i j}\right) \sqrt{T_{c, i} T_{c, j}} \quad \text { with } \quad k_{i i}=0 \\
v_{c, i j}=\frac{1}{8}\left(v_{c, i}^{\frac{1}{3}}+v_{c, j}^{\frac{1}{3}}\right)^{3}, \quad Z_{c, i j}=\frac{1}{2}\left(Z_{c, i}+Z_{c, j}\right) \\
p_{c, i j}=\frac{R_{u} T_{c, i j} Z_{c, i j}}{v_{c, i j}}
\end{gathered}
$$

with $T_{c, i}, Z_{c, i} v_{c, i}$, and $p_{c, i}$ being the pure species critical values. $\Omega_{i}$ is the species acentric factor, and $k_{i j}$ is an empirical mixing parameter. The values for hydrogen and oxygen are in Table 1.

Most data references pertain to $k_{i j}^{\prime}$, another mixing parameter related to $a_{i j}$ through

$$
k_{i j}^{\prime}=1-\frac{a_{i j}}{\sqrt{a_{i i} a_{j j}}}
$$

Replacing in Eq. (A3) $a_{i j}$ and $T_{c, i j}$ from Eqs. (A1) and (A2) yields a relationship between the parameters $k_{i j}$ and $k_{i j}^{\prime}$

$$
\left(1-k_{i j}\right)=\left(1-k_{i j}^{\prime}\right) \frac{Z_{c, i j}}{v_{c, i j}}\left(\frac{v_{c, i} v_{c, j}}{Z_{c, i} Z_{c, j}}\right)^{\frac{1}{2}}
$$

Given the lack of information regarding the values of $k_{i j}$ or $k_{i j}^{\prime}$, in the simulations, here $k_{i j}=0$.

\section{Acknowledgments}

This work was conducted at the Jet Propulsion Laboratory (JPL), California Institute of Technology, and was sponsored by the NASA Marshall Space Flight Center, under the direction of John Hutt. The computationalresources were provided by the JPL Supercomputing Center.

\section{References}

${ }^{1}$ Harstad, K., and Bellan, J., "Evaluation of Commonly Used Assumptions for Isolated and Cluster Heptane Drops in Nitrogen at All Pressures," Combustion and Flame, Vol. 127, No. 1-2, 2001, pp. 1861-1879.

${ }^{2}$ Prausnitz, J., Lichtenthaler, R., and de Azevedo, E., Molecular Thermodynamics for Fluid-Phase Equilibrium, Prentice-Hall, Upper Saddle River, NJ, 1986, pp. 442-471.

${ }^{3}$ Harstad, K., and Bellan, J., "The $D^{2}$ Variation for Isolated LOX Drops and Polydisperse Clusters in Hydrogen at High Temperature and Pressures," Combustion and Flame, Vol. 124, No. 4, 2000, pp. 535-550.

${ }^{4}$ Mayer, W., Schik, A., Schweitzer, C., and Schaffler, M., "Injection and Mixing Processes in High Pressure LOX/GH2 Rocket Combustors," AIAA Paper 96-2620, 1996.

${ }^{5}$ Mayer, W., Ivancic, B., Schik, A., and Hornung, U., "Propellant Atomization in LOX/GH2 Rocket Combustors," AIAA Paper 98-3685, 1998

${ }^{6}$ Chehroudi, B., Talley, D., and Coy, E., "Initial Growth Rate and Visual Characteristics of a Round Jet into a Sub- to Supercritical Environment of Relevance to Rocket, Gas Turbine and Diesel Engines," AIAA Paper 99$0206,1999$.

${ }^{7}$ Oschwald, M., and Schik, A., "Supercritical Nitrogen Free Jet Investigated by Spontaneous Raman Scattering." Experiments in Fluids, Vol. 27, No. 6, 1999, pp. 497-506.

${ }^{8}$ Miller, R. S., Harstad, K., and Bellan, J., "Direct Numerical Simulations of Supercritical Fluid Mixing Layers Applied to Heptane-Nitrogen," Journal of Fluid Mechanics, Vol. 436, 2001, pp. 1-39.

${ }^{9}$ Okong'o, N., and Bellan, J., "Direct Numerical Simulation of a Transitional Supercritical Mixing Layer: Heptane and Nitrogen," Journal of Fluid Mechanics (to be published)

$\checkmark{ }^{10}$ Okong'o, N., and Bellan, J., "Entropy Production of Emerging Turbulent Scales in a Temporal Supercritical n-Heptane/Nitrogen ThreeDimensional Mixing Layer," Proceedings of the Combustion Institute, Vol. 28, Pt. 1, 2000, pp. 497-504.

${ }^{11}$ Metcalfe, R. W., Orszag, S. A., Brachet, M. E., Menon, S., and Riley, J. J., "Secondary Instability of a Temporally Growing Mixing Layer," Journal of Fluid Mechanics, Vol. 184, 1987, pp. 207-243.

$\checkmark{ }^{12}$ Harstad, K., and Bellan, J., "The Lewis Number Under Supercritical Conditions," InternationalJournal of Heat andMass Transfer, Vol.42, No. 6, 1999, pp. 961-970.

${ }^{13}$ Okong'o, N., and Bellan, J., "Consistent Boundary Conditions for Multicomponent Real Gas Mixtures Based on Characteristic Waves,' Journal of Computational Physics, Vol. 176, 2002, pp. 330-344. 
${ }^{14}$ Sarman, S., and Evans, D. J., "Heat Flux and Mass Diffusion in Binary Lennard-Jones Mixtures," Physical Review A: General Physics, Vol. 45, No. 4, 1992, pp. 2370-2379.

${ }^{15}$ Harstad, K., and Bellan, J., An All-Pressure Fluid-Drop Model Applied to a Binary Mixture: Heptane in Nitrogen," International Journal of Multiphase Flow, Vol. 26, No. 10, 2000, pp. 1675-1706.

${ }^{16}$ Reid, R. C., Prausnitz, J. M., and Polling, B. E., The Properties of Gases and Liquids, 4th ed., McGraw-Hill, New York, 1987, pp. 97-108.

${ }^{17}$ Harstad, K., Miller, R. S., and Bellan, J., "Efficient High-Pressure State Equations," AIChE Journal, Vol. 43, No. 6, 1997, pp. 1605-1610.

${ }^{18}$ Harstad, K., and Bellan, J., "Isolated Liquid Oxygen Drop Behavior in Fluid Hydrogen at Rocket Chamber Pressures," International Journal of Heat and Mass Transfer, Vol. 41, No. 22, 1998, pp. 3537-3550.

${ }^{19}$ Kennedy, C. A., and Carpenter, M. H., "Several New Numerical Methods for Compressible Shear-Layer Simulations," Applied Numerical Mathematics, Vol. 14, No. 4, 1994, pp. 397-433.

${ }^{20}$ Muller, S. M., and Scheerer, D., "A Method to Parallelize Tridiagonal Solvers," Parallel Computing, Vol. 17, No. 2-3, 1991, pp. 181-188.

${ }^{21}$ Drazin, P. G., and Reid, W. H., Hydrodynamic Stability, Cambridge Univ. Press, Cambridge, England, U.K., 1981, pp. 124-153.

${ }^{22}$ Okong'o, N., and Bellan, J., "Temporal Linear Stability Analysis and Numerical Simulations of Real Gas Two- and Three-Dimensional Mixing Layers," Physics of Fluids (submitted for publication).
${ }^{23}$ Moser, R. D., and Rogers, M. M., "Mixing Transition and the Cascade to Small Scales in a Plane Mixing Layer," Physics of Fluids A, Vol. 3, No. 5, 1991,pp. 1128-1134.

${ }^{24}$ Moser, R. D., and Rogers, M. M., "The Three-Dimensional Evolution of a Plane Mixing Layer: Pairing and Transition to Turbulence," Journal of Fluid Mechanics, Vol. 247, 1993, pp. 275-320.

${ }^{25}$ Cortesi, A. B., Smith, B. L., Yadigaroglu, G., and Banerjee, S., "Numerical Investigation of the Entrainment and Mixing Processes in Neutral and Stably-Stratified Mixing Layers," Physics of Fluids, Vol. 11, No. 1, 1999, pp. 162-185.

${ }^{26}$ Miller, R. S., and Bellan, J., "Direct Numerical Simulation and Subgrid Analysis of a Transitional Droplet Laden Mixing Layer," Physics of Fluids, Vol. 12, No. 3, 2000, pp. 650-671.

${ }^{27}$ Rogers, M. M., and Moser, R. D., "Spanwise Scale Selection in Plane Mixing Layers," Journal of Fluid Mechanics, Vol. 247, 1993, pp. 321-337.

${ }^{28}$ Cortesi, A. B., Yadigaroglu, G., and Banerjee, S., "Numerical Investigation of the Formation of Three-Dimensional Structures in Stably-Stratified Mixing Layers," Physics of Fluids, Vol. 10, No. 6, 1998, pp. 1449-1473.

${ }^{29}$ Hannoun, I., Fernando, H., and List, E., "Turbulence Structure Near a Sharp Density Interface," Journal of Fluid Mechanics, Vol. 189, 1988, pp. 189-209.

M. Sichel Associate Editor 


\section{This article has been cited by:}

1. Josette Bellan. 2017. Evaluation of mixture-fraction-based turbulent-reaction-rate model assumptions for highpressure reactive flows. Combustion and Flame 179, 253-266. [Crossref]

2. Josette Bellan. 2017. Direct numerical simulation of a high-pressure turbulent reacting temporal mixing layer. Combustion and Flame 176, 245-262. [Crossref]

3. Aswin Gnanaskandan, Josette R. Bellan. Large Eddy Simulations of high pressure jets: Effect of subgrid scale modeling . [Citation] [PDF] [PDF Plus]

4. Daniel T. Banuti, Volker Hannemann, Klaus Hannemann, Bernhard Weigand. 2016. An efficient multi-fluid-mixing model for real gas reacting flows in liquid propellant rocket engines. Combustion and Flame 168, 98-112. [Crossref]

5. Abdelkrim Benmansour, Abdelkrim Liazid, Pierre-Olivier Logerais, Jean-Félix Durastanti. 2016. A 3D numerical study of LO2/GH2 supercritical combustion in the ONERA-Mascotte Test-rig configuration. Journal of Thermal Science 25:1, 97-108. [Crossref]

6. Zachary Falgout, Mattias Rahm, Zhenkan Wang, Mark Linne. 2015. Evidence for supercritical mixing layers in the ECN Spray A. Proceedings of the Combustion Institute 35:2, 1579-1586. [Crossref]

7. Christoph A. Niedermeier, Maria M. Jarczyk, Stefan Hickel, Nikolaus Adams, Michael Pfitzner. Large-Eddy Simulation of Turbulent Trans- and Supercritical Mixing . [Citation] [PDF] [PDF Plus]

8. Enrica Masi, Josette Bellan, Kenneth G. Harstad, Nora A. Okong'o. 2013. Multi-species turbulent mixing under supercritical-pressure conditions: modelling, direct numerical simulation and analysis revealing species spinodal decomposition. Journal of Fluid Mecbanics 721, 578-626. [Crossref]

9. Enrica Masi, Josette Bellan, Kenneth Harstad. Pressure Effects from Direct Numerical Simulation of High-Pressure Multispecies Mixing . [Citation] [PDF] [PDF Plus]

10. Justin Foster, Richard S. Miller. 2012. A priori analysis of subgrid mass diffusion vectors in high pressure turbulent hydrogen/oxygen reacting shear layer flames. Physics of Fluids 24:7, 075114. [Crossref]

11. Enrica Masi, Josette Bellan, Kenneth Harstad. Direct Numerical Simulation of High-Pressure Multispecies Turbulent Mixing in the Cold Ignition Regime . [Citation] [PDF] [PDF Plus]

12. Xavier Petit, Guillaume Ribert, Pascale Domingo. Large Eddy Simulation of Supercritical Fluid Injection . [Citation] [PDF] [PDF Plus]

13. Maria-Magdalena Jarczyk, Michael Pfitzner. Large Eddy Simulation of Supercritical Nitrogen Jets . [Citation] [PDF] [PDF Plus]

14. Enrica Masi, Josette Bellan. 2011. The subgrid-scale scalar variance under supercritical pressure conditions. Physics of Fluids 23:8, 085101. [Crossref]

15. Hiroshi Terashima, Soshi Kawai, Nobuhiro Yamanishi. Investigations of trans/supercritical jet mixing using a highresolution numerical method . [Citation] [PDF] [PDF Plus]

16. Nora Okong'o, Josette Bellan. 2010. Small-scale dissipation in binary-species, thermodynamically supercritical, transitional mixing layers. Computers \& Fluids 39:7, 1112-1124. [Crossref]

17. EZGI S. TASKINOGLU, JOSETTE BELLAN. 2010. A posteriori study using a DNS database describing fluid disintegration and binary-species mixing under supercritical pressure: heptane and nitrogen. Journal of Fluid Mechanics 645, 211. [Crossref]

18. M. Masquelet, S. Menon, Y. Jin, R. Friedrich. 2009. Simulation of unsteady combustion in a LOX-GH2 fueled rocket engine. Aerospace Science and Technology 13:8, 466-474. [Crossref]

19. José Sierra-Pallares, María Teresa Parra-Santos, Juan García-Serna, Francisco Castro, María José Cocero. 2009. Numerical modelling of hydrothermal flames. Micromixing effects over turbulent reaction rates. The Journal of Supercritical Fluids 50:2, 146-154. [Crossref]

20. J. Sierra-Pallares, M.T. Parra-Santos, J. García-Serna, F. Castro, M.J. Cocero. 2009. Numerical analysis of highpressure fluid jets: Application to RTD prediction in supercritical reactors. The Journal of Supercritical Fluids 49:2, 249-255. [Crossref]

21. L. Pons, N. Darabiha, S. Candel, T. Schmitt, B. Cuenot. 2009. The structure of multidimensional strained flames under transcritical conditions. Comptes Rendus Mécanique 337:6-7, 517-527. [Crossref] 
22. François-Xavier Demoulin, Stephan Zurbach, Arnaud Mura. 2009. High-Pressure Supercritical Turbulent Cryogenic Injection and Combustion: A Single-Phase Flow Modeling Proposal. Journal of Propulsion and Power 25:2, 452-464. [Crossref]

23. C. Segal, S. A. Polikhov. 2008. Subcritical to supercritical mixing. Physics of Fluids 20:5, 052101. [Crossref]

24. LAURENT C. SELLE, NORA A. OKONG'O, JOSETTE BELLAN, KENNETH G. HARSTAD. 2007. Modelling of subgrid-scale phenomena in supercritical transitional mixing layers: an a priori study. Journal of Fluid Mechanics 593. . [Crossref]

25. Joshua J. Smith, Gerald Schneider, Dmitry Suslov, Michael Oschwald, Oskar Haidn. 2007. Steady-state high pressure LOx/H2 rocket engine combustion. Aerospace Science and Technology 11:1, 39-47. [Crossref]

26. JOSETTE BELLAN. 2006. THEORY, MODELING AND ANALYSIS OF TURBULENT SUPERCRITICAL MIXING. Combustion Science and Technology 178:1-3, 253-281. [Crossref]

27. S. CANDEL*, M. JUNIPER†, G. SINGLA, P. SCOUFLAIRE, C. ROLON. 2006. STRUCTURE AND DYNAMICS OF CRYOGENIC FLAMES AT SUPERCRITICAL PRESSURE. Combustion Science and Tecbnology 178:1-3, 161-192. [Crossref]

28. NAN ZONG, VIGOR YANG*. 2006. CRYOGENIC FLUID JETS AND MIXING LAYERS IN TRANSCRITICAL AND SUPERCRITICAL ENVIRONMENTS. Combustion Science and Technology 178:1-3, 193-227. [Crossref]

29. N. Okong'o, Josette Bellan. A Priori Analysis of Subgrid-Scale Models for Large Eddy Simulations of Supercritical Binary-Species Mixing Layers . [Citation] [PDF] [PDF Plus]

30. Sridhar Palle, Christopher Nolan, Richard S. Miller. 2005. On molecular transport effects in real gas laminar diffusion flames at large pressure. Physics of Fluids 17:10, 103601. [Crossref]

31. Nan Zong, Hua Meng, Shih-Yang Hsieh, Vigor Yang. 2004. A numerical study of cryogenic fluid injection and mixing under supercritical conditions. Physics of Fluids 16:12, 4248-4261. [Crossref]

32. Joshua Smith, Dmitry Suslov, Michael Oschwald, Oskar Haidn, M. Bechle. High Pressure LOx/H2 Combustion and Flame Dynamics . [Citation] [PDF] [PDF Plus]

33. N. Okong'o, J. Bellan. 2004. Turbulence and fluid-front area production in binary-species, supercritical, transitional mixing layers. Physics of Fluids 16:5, 1467-1492. [Crossref]

34. Nora Okong', o, Josette Bellan. 2003. Real-Gas Effects on Mean Flow and Temporal Stability of Binary-Species Mixing Layers. AIAA Journal 41:12, 2429-2443. [Crossref]

35. Nora Okong'o, Josette Bellan. Helicity in Supercritical Temporal Mixing Layers . [Citation] [PDF] [PDF Plus] 